\title{
Body mass index and dental caries in children and adolescents: a systematic review of literature published 2004 to 2011
}

Merrilyn Hooley ${ }^{1 *}$, Helen Skouteris ${ }^{1}$, Cecile Boganin ${ }^{1}$, Julie Satur ${ }^{2}$ and Nicky Kilpatrick ${ }^{3,4}$

\begin{abstract}
The objective: The authors undertook an updated systematic review of the relationship between body mass index and dental caries in children and adolescents.

Method: The authors searched Medline, ISI, Cochrane, Scopus, Global Health and CINAHL databases and conducted lateral searches from reference lists for papers published from 2004 to 2011, inclusive. All empirical papers that tested associations between body mass index and dental caries in child and adolescent populations (aged 0 to 18 years) were included.

Results: Dental caries is associated with both high and low body mass index.

Conclusion: A non-linear association between body mass index and dental caries may account for inconsistent findings in previous research. We recommend future research investigate the nature of the association between body mass index and dental caries in samples that include a full range of body mass index scores, and explore how factors such as socioeconomic status mediate the association between body mass index and dental caries.
\end{abstract}

Keywords: Early childhood caries, Childhood obesity, Review, Dental caries, Obesity, Overweight

Obesity and dental caries are both multifactorial diseases that impact children's health and psychosocial development $[1,2]$. Both conditions contribute substantially to health expenditure; for example, the estimated total annual cost of obesity to Australian society in 2008 was $\$ 58.2$ billion [3], and recent estimates suggest that being overweight or obese is the third highest contributor to disability-adjusted life years (DALYs; 7.5\%) [4]. Dental disease ranks as the second most expensive disease in Australia (second to cardiovascular disease) and absorbs $6.2 \%$ of the total recurrent expenditure in health, behind hospital services (39.3\%), medical services (18.7\%), and medications (14.0\%) [4]. Obesity and dental caries share common, modifiable, influences such as diet and lifestyle. Recent national data from Sweden [5] suggest a positive correlation between dental caries and Body

\footnotetext{
*Correspondence: merrilyn.hooley@deakin.edu.au

${ }^{1}$ School of Psychology, Deakin University, 221 Burwood Highway, Burwood, Melbourne, Victoria 3125, Australia

Full list of author information is available at the end of the article
}

Mass Index (BMI), and showed that obesogenic behaviour such as snacking in early childhood predicted caries development in adolescence. BMI is widely used as a surrogate measure for obesity because it corrects for an individual's height in relation to weight, and is a commonly used indicator of can indicate nutritional status. Given that dental caries rates and BMI both measure diet-related health outcomes, an association between the two is not surprising. Changes to diet and lifestyle since the mid 1990s, such as increased affluence and access to high caloric carbohydrate-rich foods and drinks, may help to account for the rising prevalence in dental caries and obesity since that time [6,7]. However, not all studies have found a positive association between BMI and dental caries; some studies suggest that there is no relationship (for example, [8]) and others show an inverse relationship (for example, [9]).

To our knowledge there has been only one systematic review examining the relationship between obesity and dental caries [10]. This review included only seven studies published between 1984 and 2004, only five of which 
included a pediatric sample. Of the three A grade studies, one found a positive correlation between dental caries and BMI in a sample of 842 children aged 6 to 11 years [11]. Another found no correlation in a sample of over five thousand 3-year olds [12], while the third was not able to predict future dental caries experience on the basis of BMI status in more than five hundred children (aged 5 to 13 years) [13].

Since publication of this review [10] there has been increased interest in the association between dental caries and BMI. The objective of the current paper was to provide an updated review of the evidence in this emerging area of research. Our systematic review addressed the following specific questions:

1. What do studies reveal about the association between dental caries and BMI in children and adolescents?

2. What are the methodological limitations of the current approaches to investigating the development of both dental caries and obesity and what may be valuable directions for future research?

\section{Methods}

\section{Search strategy}

The authors searched Medline, Web of Science (ISI), Cochrane, Scopus, Global Health and the Cumulative Index to Nursing and Allied Health (CINAHL) libraries. Additionally, a lateral approach involving a review of reference lists in papers identified was undertaken. Search terms were Obesity OR overweight OR BMI or body mass index OR weight AND Caries or dental health OR oral health OR dmf* OR teeth decay* OR cavities OR cavities. AND Child* OR adol* OR preschool OR toddlers OR pediat* OR paediat*

We did not initially include search terms covering "malnutrition", but we found no additional studies meeting inclusion criteria after conducting a forensic search using the terms "malnutrition OR malnourish"" in addition to our original dental search terms. We included relevant non-English language studies and translated them in to English. To explore development since Kantovitz et al. article [10] the search was limited to papers published from January 2004 until June 2011. Studies were included if they satisfied the following three criteria:

1. Measured caries rates, most commonly by variations of the number of decayed (Dd), missing (Mm), filled (Ff) surfaces (Ss) or teeth (Tt) index or presence/ absence of caries (that is, DMFT/dmft $>0$ ). However studies that categorized teeth conditions (for example, levels of caries experience: frank caries, filled teeth, white spots and no caries [14]) or described dental health (for example, cavity now or ever, filling, tooth pulled, and overall dental health [15]) were also included in the review.

2. Measured some form of weight-to-height ratio to estimate body fat. This was most commonly estimated using BMI, but body fat index (DXA [16]) and Division of Nutrition, Thai Ministry of Public Health standards using weight for height in Thai children [17] were also used.

3. Assessed the relationship between dental caries and BMI in children and adolescents to age 18 years.

\section{Methodological quality}

Studies were evaluated on the basis of a number of criteria to assess quality of methodology. These criteria are summarized in Table 1 and Additional file 1 . The first criterion was representativeness of sample, and studies were ranked 1 (highest) to 4 (lowest) as follows: 1) sample involved forms of stratification or cluster sampling of countries or districts that ensured a representative range of socioeconomic strata; 2) sample represented cities or towns using some form of cluster sampling (for example, of schools); 3) sample of convenience with some randomization involved in selection of participants; and 4) sample of convenience without random selection (for example, patients of obesity clinics, dental hospitals). The second criterion was whether or not an attempt had been made to control potential confounding variables; studies were scored on a yes/no basis ( Yes = 1 ; No $=2$ ). The third criterion assessed the quality of assessment of the child weight-to-height (for example, BMI) and dental caries. Measures of child weight-to-height were scored for objective measurement using standardized equipment (score 1) or other (score 2). Scores for the quality and sensitivity of the dental examination ranged from 1 (highest) to 5 (lowest; see Table 1). Rating 1 examinations were conducted within a dental surgery with mirror and probe by a qualified dentist, dental student, or hygienist and included radiographs. Rating 2 involved dental examinations within a dental surgery with mirror and probe by a qualified dentist, dental student, or therapist/hygienist but did not include radiographs. Examinations Rated 1 or 2 were performed under optimal lighting conditions with dry field available, which improves detection rates and enabled the detection of initial caries lesions. Rating 3 involved examinations that were performed under field conditions, which typically rated caries at the cavity level. Most examinations at this level were carried out with natural lighting, blunt probe and mirror (that is, following WHO criteria), and may have been undertaken by someone other than a qualified dental professional. Rating 4 involved visual examination involving mirror only, and Rating 5 involved parent or self report. Under field conditions obtaining a dry field is difficult although some studies used cotton rolls/ pellets; a lowercase superscripted 'a' with the rating scores 
Table 1 Criteria used for rating studies

\begin{tabular}{|c|c|c|c|c|}
\hline & Sample & $\begin{array}{l}\text { Attempt made to } \\
\text { control confounds? }\end{array}$ & BMI measure & Dental caries measure \\
\hline \multirow[t]{5}{*}{1} & \multirow{5}{*}{$\begin{array}{l}\text { Stratification/cluster sampling use to obtain } \\
\text { sample representative of countries/districts }\end{array}$} & \multirow[t]{5}{*}{ Yes } & \multirow[t]{5}{*}{ Standardized } & Dental surgery \\
\hline & & & & Mirror and probe \\
\hline & & & & Optimal lighting and dry field \\
\hline & & & & Radiographs \\
\hline & & & & Dentally qualified examiner calibration \\
\hline \multirow[t]{4}{*}{2} & \multirow{4}{*}{$\begin{array}{l}\text { Some form of cluster sampling use to obtain } \\
\text { sample approximately representative of towns }\end{array}$} & \multirow[t]{4}{*}{ No } & \multirow[t]{4}{*}{ Non-standardized } & Dental surgery \\
\hline & & & & Mirror and probe \\
\hline & & & & Optimal lighting and dry field \\
\hline & & & & Dentally qualified examiner calibration \\
\hline \multirow[t]{2}{*}{3} & \multirow[t]{2}{*}{ Convenience sample with some randomization } & & & Field clinic \\
\hline & & & & Mirror and probe calibration \\
\hline \multirow[t]{2}{*}{4} & \multirow[t]{3}{*}{ Convenience sample without randomization } & & & Field clinic \\
\hline & & & & Visual inspection calibration \\
\hline 5 & & & & Parent report \\
\hline
\end{tabular}

BMI, body mass index.

of 3 or 4 denotes these studies. Calibration exercises were undertaken in all studies involving dental examinations.

The potential range of scores following assessment of methodological quality ranged from 4 to 13 . Studies scoring 4 to 5 were rated A, 6 to 7 rated B, 8 to 10 rated $\mathrm{C}$ and 11 to 13 rated $\mathrm{D}$.

\section{Results}

The flow diagram of the processing of search results is shown in Figure 1. Forty-seven papers met the selection criteria and were included in the review. However, two papers $[5,18]$ reported the same data and were combined for the purpose of this review, while two other papers $[19,20]$ reported findings of two separate studies. A total of 48 studies were therefore included, (excluded papers and reasons for exclusion are presented in Additional file 2). Only two studies were also cited in Kantovitz et al. review $[8,11]$.

Three main patterns of relationships were found between dental caries and BMI: 23 of the 48 studies found no association between BMI and dental caries, 17 found a positive relationship between BMI and dental caries, and nine found an inverse relationship. One additional study found dental caries was associated with both high- and low- BMI [21]; that is, a U-shaped pattern, and another found inconsistent patterns across age cohorts; specifically, an association between lower dental caries and high BMI in two age cohorts, and no association in four cohorts [19]. In order to explore possible explanations for these differences in patterns, the results of the 48 studies were separated and evaluated on the basis of the nature of the relationship between caries and BMI. That is, positive, negative and no association are summarized in Tables 2,3 and 4 respectively. The study finding that dental caries is associated with both high and low BMI [16] is included in both Tables 2 and 3 and the study finding no association and a negative association between dental caries and BMI depending on the age cohorts is included in Tables 3 and 4 . Table 5 summarizes the patterns that emerged within each set of studies.

Given that a number of studies used samples of convenience and special populations, (for example, hospital lists for dental extractions under general anesthetic [22] and lists for obesity programs [23]), the distribution of dental and BMI scores varies between studies. The results of these studies are of interest, but it is important to be mindful that these studies will have not tested the association between dental caries and BMI across the full range of potential scores. For this reason, sample statistics for caries and BMI measures (where provided in the studies) are included in summary tables for consideration when comparing results. The summary tables also provide information about each study's setting, design, sample, dental measures, human development index (HDI), and factors associated significantly with dental caries in childhood and adolescence to facilitate interpretation of findings across studies.

After assessing for bias, only five studies met the criteria for A rating [19,24-26], and 20 studies met the criteria for a $B$ rating (see Additional file 1). The findings of the five A-rated studies were mixed: two found that dental caries was higher in children with higher BMI $[24,25]$; one found a negative association in children 


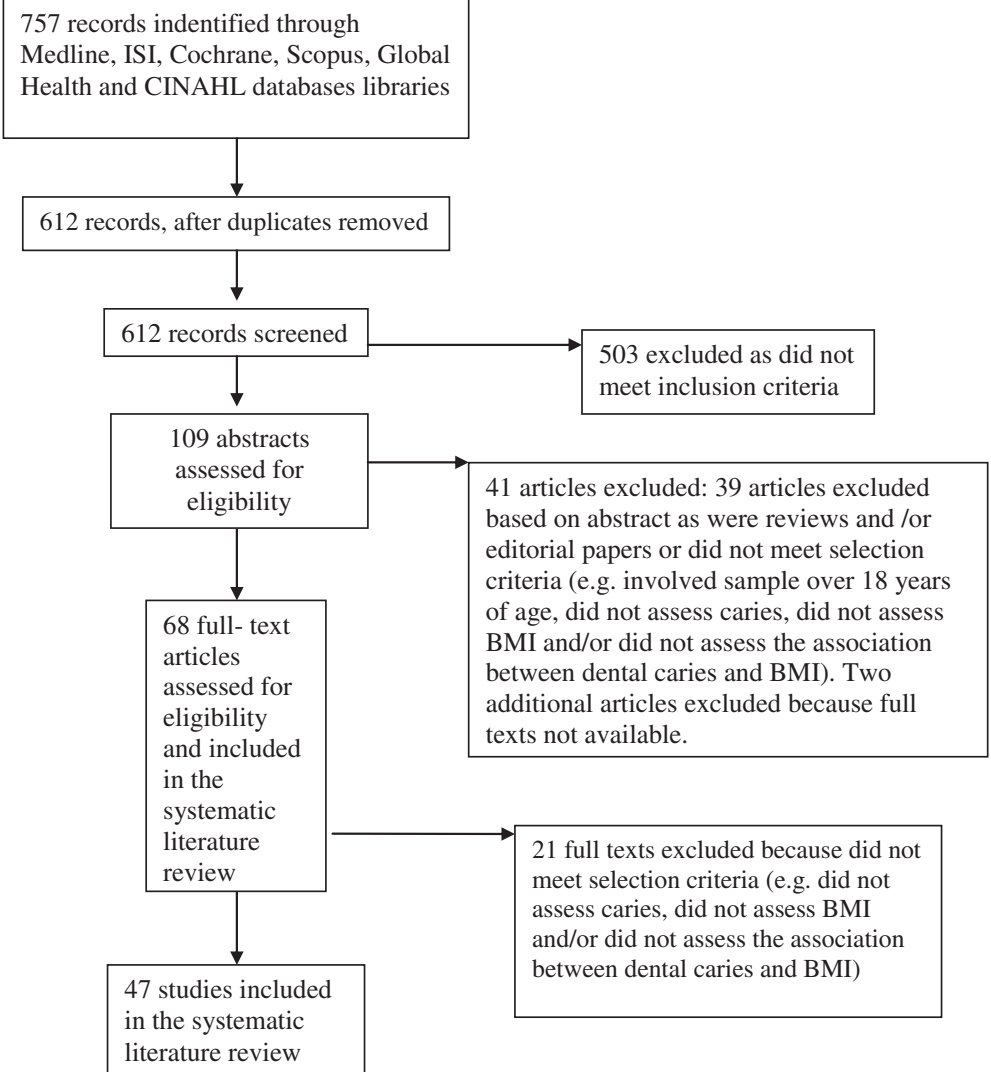

Figure 1 The flow diagram of processing of search result.

aged 6 to 11 and 12 to 18 years but no association in children aged 2 to 5 years [19]; and two found no association in children aged 2 to 17 years $[19,26]$. Four of the five studies used data collected as part of the National Health and Nutrition Examination Survey (NHANES) database, a large nationally representative sample in the United States [19,25,26], and three studies used the same 1999 to 2002 cohort [19,25,26]. Hong et al. [25], Macek and Mitola [26], and Kopycka-Kedzierawski et al. [19] analyzed the data collected from children aged 2 to 5 years in the 1999 to 2002 cohort and reported different results: positive, no association, and no association, respectively. The sample sizes between these three studies differed slightly $(1,506 ; 1,449 ; 1,719$; respectively); Hong et al. [25] excluded children with fewer than 10 teeth, which might explain the smaller sample, but no exclusion criteria are provided for the other studies. All studies found similar trends for children at risk/overweight to have higher risk of dental caries than normal-weight children. Hong et al. [25] stratified by age and found the difference to be significant in children aged 60 to 72 months whereas Macek and Mitola [26] and Kopycka-Kedzierawski et al. [19] collapsed across age and found no association. KopyckaKedzierawski et al. [19] investigated other age groups within the 1999 to 2002 cohort and found no association in children aged 6 to 18 years.

The fourth study that used the NHANES III cohort [19] found that children aged 6 to 18 years who were overweight had a reduced risk of dental caries in both permanent and deciduous teeth compared to children of normal weight. No association was found in children aged 2 to 5 years. The fifth study [24] was a large cohort study from Sweden. A prospective association between obesity at an early age (4, 5, 7, and 10 years) and dental caries at age 12 years was reported. Thus, across these five A-ranked studies, four found trends consistent with a positive association between dental caries and BMI, which were significant in only two studies. The fifth found a negative association. One important problem with all five A-ranked studies is the restricted BMI range in the samples; underweight children were significantly under-represented. Only one study reported the proportion of underweight children in their sample (4.2\%; [25]), and no studies included underweight as a BMI category. This is problematic for two reasons. First, the association between BMI and dental caries was inadequately tested because the association was not tested across the full range of BMI scores, and second, it must be concluded 
Table 2 Positive association between dental caries and BMI

Authors Country Design N $\quad$ Age Sampling

Alm (2008) Sweden Prospective 402 at age

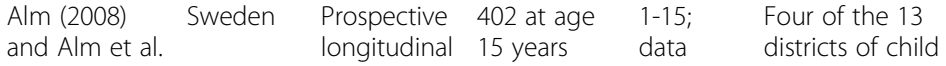

1-15; $\quad$ Four of the 13

(2008)

analysed welfare centres in

15 years of Jonkoping

\section{Dental}

HDI Dental measure

Significant cariogenic

Sample demographics

risk factors

Initial caries: $86 \%$ of total

$D_{\text {ia }}$ (Initial caries) Higher approximal dental

$D_{\text {ma }}$ (Manifest caries) caries in overweight and

$\mathrm{D}_{\mathrm{i}+\mathrm{m}} \mathrm{FA}$ or $\mathrm{DF}_{\mathrm{a}}$ (Total obese adolescents than

approximal caries

normal and underweight.

Caries experience at 15 years predicted by early childhood

caries experience at age 3,

plaque on incisors at age 1

year, infrequency of tooth

brushing with fluoride

toothpaste, consumption of

caries-risk snacks more than

three times a day at age 1

$<$ once per week at age 3

years, parents were born

abroad, parents' poor

attitudes to dental health

caries experience

Approximal caries prevalent

in $67 \%$ of $15 \mathrm{yr}$ old children

Mn Di+mFa at 15 yrs

Overall sample $=3.21(\mathrm{SD}=3.95)$

Underweight $=2.94(\mathrm{SD}=3.62)$

Normal weight $=4.64(\mathrm{SD}=5.15)$

Overweight $=4.18(\mathrm{SD}=5.14)$

Obese $=6.29(\mathrm{SD}=5.04)$

$\mathrm{Mn}$ DmFa at 15 yrs

Overall sample 0.42 ( $S D=1.13$ )

Underweight $=0.35(\mathrm{SD}=-0.89)$

Normal weight $=0.83(\mathrm{SD}=1.93)$

Overweight $=0.84(\mathrm{SD}=2.02)$

Obese $=0.79(\mathrm{SD}=1.63)$

\section{BMI:}

and psychosocial factors

\begin{tabular}{lllll}
\hline $\begin{array}{l}\text { Alm et al. Sweden } \\
\text { (2011) }\end{array}$ & L \&CS & Time 1 & $1-15$ & Four of the 13 \\
& & (aged 3): & districts of child \\
& 525 Time 2 & welfare centres \\
& (aged 6): & in the Municipality \\
& 506 Time 3 & of Jonkoping \\
& (aged 15): & \\
& 402 &
\end{tabular}

10

Caries (Initial and

At 3 years: No association

Overweight: 16\%

Obese: $4 \%$

Low-normal weight: $84 \%$

\section{BMI:}

manifest) prevalence: At 6 years: Higher prevalence

def in children 3 and of manifest caries in

6 years

overweight/obese

At 15 years: Higher rates of

Manifest caries

proximal caries/fillings in

prevalence: $\left(D_{m} a\right) \quad$ overweight/obese

3 years:

Normal weight: $86 \%$

Overweight: 12\%

Obese: 3\%

6 years:

Normal weight: 82\%

Overweight: 14\%

Obese: $4 \%$

15 years:

Normal weight: $84 \%$

Overweight: 12\%

Obese: $4 \%$

\begin{tabular}{|c|c|c|c|c|c|c|c|c|c|c|}
\hline $\begin{array}{l}\text { Bailleul- } \\
\text { Forestier } \\
\text { et al. (2007) }\end{array}$ & France & CC & 82 & $12-18$ & $\begin{array}{l}\text { Case-match control: } \\
\text { Treatment program } \\
\text { for severe obesity } \\
\text { matched for age, } \\
\text { gender and parental } \\
\text { socio-occupation }\end{array}$ & $\begin{array}{l}3 \text { Cavity } \\
\text { level }\end{array}$ & 20 & $\begin{array}{l}\text { DMFT - ordinal } \\
\text { ranking 1-8 }\end{array}$ & $\begin{array}{l}\text { Significant association } \\
\text { between high dental caries } \\
\text { and increased obesity. }\end{array}$ & $\begin{array}{l}\text { Caries prevalence: } \\
\text { Obese - 90\% } \\
\text { Control - } 80 \% \\
\text { Mn DMFT } \\
\text { Obese } 6.9(\mathrm{SD}=4.1) \\
\text { Control: } 4.3(\mathrm{SD}=3.5) \\
\text { Mn BMI: } \\
\text { Obese group: } 40.6(\mathrm{SD}=7.3) \\
\text { Control: } 19.8(\mathrm{SD}=2.1)\end{array}$ \\
\hline $\begin{array}{l}\text { Costacurta et } \\
\text { al. (2011) }\end{array}$ & Italy & CS & 107 & $6-12$ & $\begin{array}{l}\text { Paediatric } \\
\text { Dentistry Unit } \\
\text { of PTV Hospital, } \\
\text { University of } \\
\text { Rome "Tor Vergata" }\end{array}$ & 1 & 24 & Dmft/DMFT & $\begin{array}{l}\text { Child physical status } \\
\text { measured as } \\
\text { (i) Fat Mass\% (FM; using } \\
\text { Dual energy X-ray), and } \\
\text { (ii) BMl }\end{array}$ & $\begin{array}{l}\text { Caries Prevalence: } 83.18 \% \\
\text { Total Mn dmft }=2.07(\mathrm{SD}=1.21) \\
\text { by FM\% (WHO cut offs) } \\
\text { Underweight }=2(\mathrm{SD}=0.61) \\
\text { Normal weight }=1.2(\mathrm{SD}=0.36)\end{array}$ \\
\hline
\end{tabular}




\begin{tabular}{|c|c|c|c|c|c|c|c|c|c|c|}
\hline & & & & & & & & & $\begin{array}{l}\text { Children with higher body } \\
\text { fat mass (FM\%) had higher } \\
\text { DMFT/dmft rates than } \\
\text { those with normal FM, but } \\
\text { comparable dmft/DMFT rates } \\
\text { with underweight children } \\
\text { using FM\%-DXA } \\
\text { No association found using } \\
\text { BMI (McCarthy or WHO } \\
\text { cut-offs) as obesity estimate }\end{array}$ & $\begin{array}{l}\text { Overweight }=1.95(\mathrm{SD}=0.25) \\
\text { Obese = } 2.40(\mathrm{SD}=0.52) \\
\text { Total Mn DMFT }=2.55(\mathrm{SD}=2.02) \\
\text { by FM\% (WHO cut offs) } \\
\text { Underweight }=3.6(\mathrm{SD}=2.7) \\
\text { Normal weight }=1.88(\mathrm{SD}=1.48) \\
\text { Overweight }=1.74(\mathrm{SD}=1.68) \\
\text { Obese }=3.10(\mathrm{SD}=2.11) \\
\text { Physical status } \\
\text { (FM\% -Who cut-offs) } \\
\text { Underweight }=4 \% \\
\text { Healthy weight }=22 \% \\
\text { Overweight }=21 \% \\
\text { Obese }=51 \%\end{array}$ \\
\hline $\begin{array}{l}\text { Gerdin et al. } \\
(2008)\end{array}$ & Sweden & L & 2303 & 4-12 & $\begin{array}{l}\text { Retrospective archival } \\
\text { study of children in } \\
\text { single county }\end{array}$ & $\begin{array}{l}1 \\
\text { manifest } \\
\text { caries } \\
\text { only }\end{array}$ & 10 & $\begin{array}{l}\text { Deft (6 yrs) DFT } \\
\text { (10-12 yrs) DFSa } \\
\text { (approximal } \\
\text { surfaces of } \\
\text { permanent teeth) }\end{array}$ & $\begin{array}{l}\text { Dental caries higher } \\
\text { prevalence in obese than } \\
\text { non-obese } \\
\text { At 12-years of age, children } \\
\text { who had a history of obesity } \\
\text { at 4-yrs of age had higher } \\
\text { rates of caries than children } \\
\text { who had history of healthy } \\
\text { weight at 4-years of age. } \\
\text { Obese/overweight children at } \\
4 \text { yrs old and who remained } \\
\text { overweight / obese at } 5,7 \\
\text { and } 10 \text { years of age had } \\
\text { significantly more approximal } \\
\text { carious surfaces (DFSa) than } \\
\text { normal weight children in all } \\
\text { age groups } \\
\text { Overweight / obese children } \\
\text { at } 4 \text { yrs old but with normal } \\
\text { weight at } 5,7 \text { and } 10 \text { yrs of } \\
\text { age had significantly fewer } \\
\text { approximal carious surfaces } \\
\text { than children with normal } \\
\text { weight from } 4 \text { to } 10 \text { years of } \\
\text { age } \\
\text { Gender: Girls at } 10 \text { and } 12 \\
\text { yrs old had more caries } \\
\text { affected teeth than boys } \\
\text { SES: Caries prevalence decreased } \\
\text { with increasing SES }\end{array}$ & $\begin{array}{l}\text { Caries Prevalence: } \\
\text { Age 6=31\% } \\
\text { Age 10=23.1\% } \\
\text { Age 12=32\% } \\
\text { Mn Dft/DFT: } \\
\text { Age 6= 3.8 } \\
\text { Age 10=1.9 } \\
\text { Age 12 = 2.1 } \\
\text { BMl: } \\
\text { Age 4: } \\
\text { Normal BMI= } 87.2 \% \\
\text { Overweight= } 10.8 \% \\
\text { Obese= } 2.0 \% \\
\text { Age 5: } \\
\text { overweight/obese = 14.2\% } \\
\text { Age 6: Not reported } \\
\text { Age 7: overweight/obese = } \\
\text { 17.1\% } \\
\text { Age 10: } \\
\text { Overweight: } 17.5 \% \\
\text { Obese= } 4.3 \%\end{array}$ \\
\hline
\end{tabular}


Table 2 Positive association between dental caries and BMI (Continued)

\begin{tabular}{|c|c|c|c|c|c|c|c|c|c|c|}
\hline $\begin{array}{l}\text { Hilgers et al. } \\
\text { (2006) }\end{array}$ & USA & CS & $\begin{array}{l}178 \text { sample } \\
\text { of } \\
\text { convenience }\end{array}$ & 8-11 & $\begin{array}{l}\text { Convenience sample } \\
\text { - participants of } \\
\text { dental treatment } \\
\text { program Smile } \\
\text { Kentucky - dental } \\
\text { needs with no dental } \\
\text { insurance }\end{array}$ & 1 & 4 & $\begin{array}{l}\text { Interproximal } \\
\text { caries in primary } \\
\text { and permanent } \\
\text { molars Ordinal } \\
\text { ranking, 1=incipient, } \\
2=\text { dentine } \\
\text { involvement, } 3= \\
\text { pulpal involvement; } \\
4=\text { nonrestorable/ } \\
\text { missing } \\
\text { Severity index } \\
\text { calculated by } \\
\text { averaging primary } \\
\text { and permanent } \\
\text { tooth scores } \\
\text { (c-avg and C-avg) }\end{array}$ & $\begin{array}{l}\text { Higher permanent molar } \\
\text { caries average associated } \\
\text { with higher BMl }\end{array}$ & $\begin{array}{l}\text { C-avg (severity measure } \\
\text { for permanent teeth) } \\
\text { ranged from } 0 \text { to } 4.0 \\
\text { Low BMI:C-avg }=0.08(0.06) \\
\text { Normal BMl: C-avg } 0.19(0.05) \\
\text { High BMl: C-avg=0.51 (0.09) } \\
\text { BMI } \\
\quad \text { Ranged from 11.43-35.7 }\end{array}$ \\
\hline $\begin{array}{l}\text { Hong et al. } \\
\text { (2008) }\end{array}$ & USA & CS & 1507 & $2-6$ & NHANES (1999-2002) & 2 & 4 & Dft $0,1-5,>5$ teeth & $\begin{array}{l}\text { Higher caries rates } \\
\text { significantly associated with } \\
\text { higher BMI in } 5-6 \text { yr olds and } \\
\text { in Hispanic and non-hispanic } \\
\text { blacks } \\
\text { S-ECC: Logistic regression } \\
\text { found BMl did not predict of } \\
\text { caries experience but Age } \\
\text { and poverty index did }\end{array}$ & $\begin{array}{l}\text { Caries prevalence } \mathbf{4 2} \% \\
\text { Mn dft 1.79(.09) } \\
\text { BMI } \\
\text { Mn BMI } 16.2(.01) \\
\text { Underweight } 4.2 \% \\
\text { Normal } 73.9 \% \\
\text { At risk } 11.3 \% \\
\text { Overweight } 10.6 \%\end{array}$ \\
\hline $\begin{array}{l}\text { Ismail et al. } \\
\text { (2009) }\end{array}$ & USA & $L \& C S$ & 788 & $0-5$ & $\begin{array}{l}\text { A two-stage area } \\
\text { probability selection } \\
\text { of representative } \\
\text { sample of low- } \\
\text { income African- } \\
\text { American in Detroit } \\
\text { Michigan. Dyads } \\
\text { tested in 2002-3 and } \\
2004-5\end{array}$ & 2 & 4 & $\begin{array}{l}\text { Non-cavitated } \\
\text { lesions: (d1-2) } \\
\text { Cavitated/dentinal } \\
\text { lesions: (d3-6) } \\
\text { Filled lesions (f); } \\
\text { missing lesions (m) } \\
\text { d3-6mfs; d1-6mfs }\end{array}$ & $\begin{array}{l}\text { Higher caries (dmft:1-6) } \\
\text { associated with higher } \\
\text { weight-for-age. For d1-6mfs: } \\
\text { higher consumption of soda } \\
\text { drinks, older child age, higher } \\
\text { weight-for-age, visiting a } \\
\text { dentist for treatment, higher } \\
\text { baseline caries level of the } \\
\text { child and caregiver, fatalistic } \\
\text { belief of the caregiver, and } \\
\text { living in relatively disadvan } \\
\text { taged low-income neighbour } \\
\text { hood. }\end{array}$ & $\begin{array}{l}\text { Almost } 25 \% \text { of children } \\
\text { had low weight for age }\end{array}$ \\
\hline $\begin{array}{l}\text { Marshall et al. } \\
\text { (2007) }\end{array}$ & USA & $L$ & 427 & $1-11$ & IOWA fluoride study & $\begin{array}{l}\text { 3- cavity } \\
\text { level }\end{array}$ & 4 & $\begin{array}{l}\text { Caries experience } \\
\text { dichotomised }=/>0\end{array}$ & $\begin{array}{l}\text { Caries experience associated } \\
\text { with: At-risk of overweight } \\
\text { lower family income Less } \\
\text { educated parent Heavier } \\
\text { mothers Higher soda pop } \\
\text { intake by age } \\
\text { Final prediction } \\
\text { model: mother's education } \\
\text { and 'at risk of overweight' }\end{array}$ & $\begin{array}{l}\text { Caries Prevalence: 31\% } \\
\text { BMI: } \\
\text { Underweight 3\% } \\
\text { Normal 72\% } \\
\text { At risk overweight 19\% } \\
\text { Overweight 5\% }\end{array}$ \\
\hline
\end{tabular}


Table 2 Positive association between dental caries and BMI (Continued)

\begin{tabular}{|c|c|c|c|c|c|c|c|c|c|c|}
\hline $\begin{array}{l}\text { Martinez- } \\
\text { Sotolongo \& } \\
\text { Martinez-Brito } \\
\text { (2010) }\end{array}$ & Cuba & CS & 649 & $8-13$ & $\begin{array}{l}\text { The primary schools } \\
\text { and one seconday } \\
\text { school in Santa } \\
\text { Marta, Varadero }\end{array}$ & $\begin{array}{l}3 \text { unclear } \\
\text { whether } \\
\text { initial } \\
\text { caries } \\
\text { included }\end{array}$ & 51 & $\mathrm{DFT} / \mathrm{dft}$ & $\begin{array}{l}\text { Higher dental caries } \\
\text { associated with higher BMI }\end{array}$ & $\begin{array}{l}\text { Caries Prevalence: } \\
\text { Normal weight: } 41.77 \% \\
\text { Obese: } 89.7 \% \\
\text { BMI: } \\
\text { Obese: } 37.3 \% \\
\text { Normal weight; } 62.71 \% \\
\text { Underweight: } 0 \%\end{array}$ \\
\hline $\begin{array}{l}\text { Modeer et al. } \\
\text { (2010) }\end{array}$ & Sweden & CC & 130 & $10.3-18.3$ & $\begin{array}{l}\text { Case-matched } \\
\text { control study }\end{array}$ & 1 & 10 & $\begin{array}{l}\text { Decayed surfaces } \\
\text { DS(>0) DMFT } \\
\text { indices }\end{array}$ & $\begin{array}{l}\text { BMI-sds associated with } \\
\text { Decayed surface (DS }>0) \text { OR } \\
\text { 1.31 (unadjusted): Age, gender, } \\
\text { chronic disease, medication, } \\
\text { salivary flow, bleeding on } \\
\text { probing visible plaque index, } \\
\text { tooth-brushing infrequency } \\
\text { (evening and morning), } \\
\text { parental country of birth, and } \\
\text { educational level No } \\
\text { association between BMI- } \\
\text { sds and DFT/DMFT }\end{array}$ & $\begin{array}{l}\text { Caries prevalence not provided } \\
\text { Obese: } \\
\text { Mean dmfs:2.2 ( } \mathrm{SD}=2.8) \\
\text { Mean dft:2.2 ( } \mathrm{SD}=2.5) \\
\text { Mean ds:0.7 ( } \mathrm{SD}=1.6) \\
\text { Control: } \\
\text { Mean dmfs: } 2.6(\mathrm{SD}=3.8) \\
\text { Mean dft: } 2.1(\mathrm{SD}=2.7) \\
\text { Mean ds:0.1 (SD }=.4) \\
\text { Mean BMI: } \\
\text { Obese: } 36.8(\mathrm{SD}=5.8) ; \\
\text { Control: } 19.7(\mathrm{SD}=2.4)\end{array}$ \\
\hline $\begin{array}{l}\text { Reifsnider et } \\
\text { al. (2004) }\end{array}$ & $\begin{array}{l}\text { USA } \\
\text { (Mexican- } \\
\text { American } \\
\text { sample) }\end{array}$ & $L$ & 104 & $1-2$ & $\begin{array}{l}\text { Obese babies } \\
\text { enrolled in Special } \\
\text { Supplemental } \\
\text { Nutrition Program for } \\
\text { Women, Infants and } \\
\text { Children }\end{array}$ & 4 & 4 & $\begin{array}{l}\text { Ordinal: Caries } \\
\text { free }=0, \text { white } \\
\text { spots }=1, \text { filling }=2, \\
\text { frank caries }=3\end{array}$ & $\begin{array}{l}\text { Higher dental caries associated } \\
\text { with higher BMl } \\
\text { Dental insurance, transpor } \\
\text { tation issues, lack of know } \\
\text { ledge of where to obtain } \\
\text { dental care for children and } \\
\text { mother's perception of the } \\
\text { condition of her children's } \\
\text { teeth }\end{array}$ & BMI: 20.3 (SD = not provided) \\
\hline $\begin{array}{l}\text { Sharma \& } \\
\text { Hedge (2009) }\end{array}$ & India & CS & $\begin{array}{l}500 \text { sample } \\
\text { of } \\
\text { convenience }\end{array}$ & $8-12$ & $\begin{array}{l}\text { Department of } \\
\text { Pedodontics and } \\
\text { Preventive Children } \\
\text { Dentistry, A.B Shetty } \\
\text { Memorial Institute of } \\
\text { Dental Sciences, } \\
\text { Mangalore }\end{array}$ & $\begin{array}{l}2- \\
\text { whether } \\
\text { initial } \\
\text { caries } \\
\text { was } \\
\text { included } \\
\text { is not } \\
\text { specified }\end{array}$ & 134 & DMFS/dmfs & $\begin{array}{l}\text { Higher rates of dental caries } \\
\text { (DMFS) in overweight and } \\
\text { obese children than normal } \\
\text { weight children. } \\
\text { Underweight children had } \\
\text { significantly higher DMFS } \\
\text { rates (but not dfs) than normal } \\
\text { weight, overweight and obese } \\
\text { children. } \\
\text { Overweight children had } \\
\text { higher preference for fatty } \\
\text { and sweet foods than normal } \\
\text { weight children }\end{array}$ & $\begin{array}{l}\text { Mean DFMS (SD not provided): } \\
\text { Underweight: } 3.11 \text { Normal } \\
\text { weight: } 1.58 \\
\text { Overweight: } 2.48 \\
\text { Obese: } 2.85 \\
\text { Mean dfs: } \\
\text { Underweight: } 2.00 \\
\text { Normal weight: } 2.14 \\
\text { Overweight: } 4.79 \\
\text { Obese: } 3.25 \\
\text { BMI: } \\
\text { Underweight } 8.6 \% \\
\text { Normal weight } 58.4 \% \\
\text { Overweight or at risk for } \\
\text { overweight } 22.2 \% \\
\text { Obese } 10.8 \%\end{array}$ \\
\hline
\end{tabular}


Table 2 Positive association between dental caries and BMI (Continued)

\begin{tabular}{|c|c|c|c|c|c|c|c|c|c|c|}
\hline $\begin{array}{l}\text { Vazquez-Nava } \\
\text { et al. (2010) }\end{array}$ & Mexico & CS & 1160 & $4-5$ & $\begin{array}{l}\text { Cohort study of } \\
\text { children in three } \\
\text { cities, Tampico, } \\
\text { Madero, and Altamira } \\
\text { in Mexico }\end{array}$ & $\begin{array}{l}3^{*} \text { with } \\
\text { white } \\
\text { spots } \\
\text { coded as } \\
\text { initial } \\
\text { caries }\end{array}$ & 57 & deft, defs & $\begin{array}{l}\text { Overweight and at-risk } \\
\text { overweight children had } \\
\text { higher caries prevalence than } \\
\text { children who were not } \\
\text { overweight } \\
\text { Caries also associated with } \\
\text { sugar consumption, bottle } \\
\text { feeding, smoking at home } \\
\text { and tooth brushing } \leq \text { once } \\
\text { per day }\end{array}$ & $\begin{array}{l}\text { Caries Prevalence } 17.9 \% \\
19.6 \% \text { boys } \\
16.4 \% \text { girls } \\
\text { Mn deft: } \\
\text { Total sample = } 1.08(2.33) \\
\text { Normal weight= } 0.70(1.94) \\
\text { At -risk overweight }=1.50(2.57) \\
\text { Overweight = } 1.51(2.71) \\
\text { Mn defs: } \\
\text { Total sample = } 1.43(3.28) \\
\text { Normal weight }=0.93(2.64) \\
\text { At-risk overweight }=1.95(3.49) \\
\text { Overweight= } 2.04(3.97) \\
\text { BMl: } \\
\text { Normal weight: } 53.7 \% \\
\text { At-risk overweight: } 14.2 \% \\
17.1 \% \text { girls } \\
11.3 \% \text { boys } \\
\text { Overweight: } 32.1 \% \\
\end{array}$ \\
\hline $\begin{array}{l}\text { Willershausen } \\
\text { et al. (2007a) }\end{array}$ & Germany & $\mathrm{CS}$ & 1290 & $6-11$ & $\begin{array}{l}5 \text { elementary schools } \\
\text { in a medium sized } \\
\text { city }\end{array}$ & 2 & 9 & DF-T df-t & $\begin{array}{l}\text { Higher rates of dental caries } \\
\text { associated with higher BMI in } \\
\text { both primary and permanent } \\
\text { dentition } \\
\text { Higher rates of caries also } \\
\text { associated with Age (older), } \\
\text { Gender (M), daily consumption } \\
\text { of sweets }\end{array}$ & $\begin{array}{l}\text { Caries Prevalence: } 61.4 \% \\
\text { Mn df-t } \\
\text { Underweight= } 1.43(2.02) \\
\text { Normal weight= } 1.82(2.41) \\
\text { Overweight= } 2.3(2.75) \\
\text { Obese= } 2.21(2.8) \\
\text { Mn DF-T } \\
\text { Underweight= }=.38(1.28) \\
\text { Normal weight= } 0.53(1.2) \\
\text { Overweight= } 0.85(1.4) \\
\text { Obese= } 0.82(1.3) \\
\text { BMI: } \\
\text { Underweight 3.6\% } \\
\text { Normal weight } 74.7 \% \\
\text { Overweight, } 11.8 \% \\
\text { Obese } 9.7 \%\end{array}$ \\
\hline $\begin{array}{l}\text { Willershausen } \\
\text { et al. (2007b) }\end{array}$ & Germany & CS & 2071 & $6-10$ & $\begin{array}{l}5 \text { elementary schools } \\
\text { in Mainz }\end{array}$ & 2 & 9 & $\begin{array}{l}\text { DF-T df-t } \\
\text { Dichotomised } \\
\text { DF-t/df-t }>0\end{array}$ & $\begin{array}{l}\text { Higher rates of dental caries } \\
\text { associated with higher BMI in } \\
\text { both permanent and } \\
\text { deciduous dentitions; Age }\end{array}$ & $\begin{array}{l}\text { Caries Prevalence 54.1\% } \\
\text { Mn df-t + DF-T } \\
\text { ranged from 1.4- } 2.6 \\
\text { Mn df-t +DF-T } \\
\text { Underweight }=1.67 \\
\text { Normal weight }=2.15 \\
\text { Overweight = } 2.64 \\
\text { Obese }=2.7 \\
\text { BMI: } \\
\text { Underweight } 6.8 \% \\
\text { Normal } 76.4 \% \\
\text { Overweight } 10.5 \% \\
\text { Obese } 6.3 \%\end{array}$ \\
\hline
\end{tabular}


Table 2 Positive association between dental caries and BMI (Continued)

\begin{tabular}{|c|c|c|c|c|c|c|c|c|c|c|}
\hline $\begin{array}{l}\text { Willershausen } \\
\text { et al. (2004) }\end{array}$ & Germany & CS & 842 & $6-11$ & $\begin{array}{l}4 \text { elementary schools } \\
\text { of diverse social } \\
\text { background from } \\
\text { single medium sized } \\
\text { city }\end{array}$ & 2 & 9 & DF-T df-t & $\begin{array}{l}\text { Higher rates of dental caries } \\
\text { associated with higher BMI in } \\
\text { both permanent and deciduous } \\
\text { Gender (slightly higher cavities } \\
\text { prevalence in boys, particularly } \\
\text { in the DF-T-index and if } \\
\text { overweight. }\end{array}$ & $\begin{array}{l}\text { Caries prevalence: } 63 \% \\
\text { Mn DF-T } \\
\text { Normal weight }=0.57 \\
\text { Overweight }=0.91 \\
\text { Obese }=0.88 \\
\text { Mn df-t } \\
\text { Normal weight }=2.08 \\
\text { Overweight }=2.48 \\
\text { Obese }=2.23 \\
\text { BMl: } \\
\text { Underweight 2.1\% } \\
\text { Normal weight } 71.7 \% \\
\text { Overweight 12.0\% } \\
\text { Obese } 13.3 \%\end{array}$ \\
\hline
\end{tabular}


Table 3 Negative association between caries and BMI (higher caries associated with lower BMI)

\begin{tabular}{|c|c|c|c|c|c|c|c|c|c|c|}
\hline Authors & Country & Design & $\mathbf{N}$ & Age & Sampling & $\begin{array}{l}\text { Dental } \\
\text { rating }\end{array}$ & $H D I^{*}$ & Dental measure & $\begin{array}{l}\text { Significant cariogenic risk } \\
\text { factors }\end{array}$ & Sample demographics \\
\hline $\begin{array}{l}\text { Benzian et al. } \\
\text { (2011) }\end{array}$ & Philippines & CS & 1951 & $\begin{array}{l}11- \\
13\end{array}$ & $\begin{array}{l}\text { Stratified cluster } \\
\text { sampling (68 public } \\
\text { schools) }\end{array}$ & $\begin{array}{l}3^{*} \text { at } \\
\text { dentinal/ } \\
\text { cavity } \\
\text { level }\end{array}$ & 112 & $\begin{array}{l}\text { DMFT+dmft } \\
\text { index } \\
\text { PUFA+pufa } \\
\text { (odontogenic } \\
\text { infections) index } \\
\text { Also categorised: } \\
\text { dmft+DMFT }>0 \\
\text { PUFA/+pufa }<1 \text {; } \\
>1\end{array}$ & $\begin{array}{l}\text { Higher DMFT+dmft rates in } \\
\text { underweight than normal } \\
\text { weight. } \\
\text { Higher PUFA+pufa index in } \\
\text { underweight than normal } \\
\text { weight. } \\
\text { Final regression model } \\
\text { found } \\
\text { PUFA+pufa >0, Gender (M), } \\
\text { No. } \\
\text { siblings (>4) more likely to } \\
\text { have low BMl } \\
\text { PUFA/pufa index better } \\
\text { predictor or } \\
\text { BMl than DMFT/dmft } \\
\text { Children with caries involving pulp } \\
\text { or with odontogenic infection had } \\
\text { increased risk of low BMl }\end{array}$ & $\begin{array}{l}\text { Caries Prevalence: } 82.3 \% \\
\text { Mn DMFT+dmft = } 3.12 \\
\text { Odontogenic infections: } \\
55.7 \% \\
\text { Mn PUFA+pufa }=1.15 \\
\text { BMl: } \\
\text { Above normal: } 1 \% \\
\text { Below normal: } 27.1 \%\end{array}$ \\
\hline $\begin{array}{l}\text { Cameron et al. } \\
\text { (2006) }\end{array}$ & Scotland & CS & $\begin{array}{l}165 \text { children } \\
\text { with severe } \\
\text { dental } \\
\text { decay }\end{array}$ & $3-11$ & $\begin{array}{l}\text { Restricted: Children } \\
\text { attending for } \\
\text { extraction under GA }\end{array}$ & 2 & 28 & $\begin{array}{l}\text { dmft dentine } \\
\text { caries }\end{array}$ & $\begin{array}{l}\text { Higher dmft in underweight } \\
\text { children } \\
\text { Higher dental caries also associated } \\
\text { with Carstairs index (measure of } \\
\text { social deprivation) }\end{array}$ & $\begin{array}{l}\text { Caries: Mn dmft } 7.9(S D=3.5) \\
\text { BMl: stats not provided } \\
\quad 71 \% \text { children socially deprived }\end{array}$ \\
\hline Floyd (2009) & Taiwan & CS & 577 & 6 & $\begin{array}{l}\text { Two schools (affluent } \\
\text { and less affluent) in } \\
\text { Taipei }\end{array}$ & 3 & 24 & def & $\begin{array}{l}\text { Higher caries (def) associated with } \\
\text { lower BMl in less affluent group } \\
\text { but not in affluent group. } \\
\text { Housing and parental occupation } \\
\text { for the less affluent sample were } \\
\text { marginally associated ( } p=0.061 \text { and } \\
0.071 \text { respectively) }\end{array}$ & $\begin{array}{l}\text { Mn def: } \\
\text { Less affluent group }= \\
6.81(\mathrm{SD}=3.66) \\
\text { Affluent group }=4.78(\mathrm{SD}=3.12) \\
\text { BMI : } \\
\text { Less affluent group }=14.04 \\
\text { (SD=1.33) } \\
\text { Affluent Group }=14.80 \\
\text { (SD=1.83) }\end{array}$ \\
\hline $\begin{array}{l}\text { Kopycka- } \\
\text { Kedzierawski et } \\
\text { al. (2008) a }\end{array}$ & USA & CS & 10180 & $2-18$ & $\begin{array}{l}\text { NHANES III (1988-1994) } \\
\text { Nationally } \\
\text { representative sample }\end{array}$ & 2 & 4 & $\begin{array}{l}\text { DMFS and dfs } \\
\text { dichotomised as } \\
\text { either having } \\
\text { caries experience } \\
\text { or not } \\
\text { Dfs and DFS in } \\
\text { children aged 2-11 } \\
\text { years were } \\
\text { estimated }\end{array}$ & $\begin{array}{l}\text { Age 6-11 years: } \\
\text { Higher caries risk in Normal-weight } \\
\text { children than: } \\
\text {-at-risk or overweight (decid) } \\
\text {-overweight (Perm) } \\
\text { Age } 12-18 \text { years: } \\
\text { Higher caries risk in Normal-weight } \\
\text { children than: } \\
\text { - overweight (Perm) } \\
\text { Multiple logistic regression: } \\
6-11 \text { years of age, } \\
\text { poverty, low level of education of }\end{array}$ & $\begin{array}{l}\text { Caries prevalence (\%): } \\
\text { 6-11yrs old (Primary dentition) } \\
\text { Overall:49.5 (1.6) } \\
\text { Overweight= } 40.6(4.7) \\
\text { At risk = 45.5 (4.6) } \\
\text { Normal weight= } 51.4(1.6) \\
\text { Underweight }=\text { not reported } \\
\text { 6-11yrs old (Permanent dentition) } \\
\text { Overall:25.9(1.7) } \\
\text { Overweight= 17.6 (2.9) } \\
\text { At risk = 29.9 (4) } \\
\text { Normal weight= } 26.5(1.7)\end{array}$ \\
\hline
\end{tabular}




\begin{tabular}{|c|c|c|c|c|c|c|c|c|c|c|}
\hline & & & & & & & & & $\begin{array}{l}\text { household head and serum cotinine } \\
\text { levels significantly associated with } \\
\text { increased risk of caries experience } \\
\text { in the primary dentition. } \\
\text { Low level of education of } \\
\text { household head, blood lead level } \\
\text { above the median and other race/ } \\
\text { ethnicity associated with increased } \\
\text { risk of having caries experience in } \\
\text { permanent dentition } \\
\text { 12-18 years of age: Low level of } \\
\text { education of household head and } \\
\text { time since last dental visit also } \\
\text { associated with increased risk of } \\
\text { caries experience in permanent } \\
\text { dentition }\end{array}$ & $\begin{array}{l}\text { Underweight }=\text { not reported } \\
\text { 12-18yrs (Permanent dentition) } \\
\text { Overall: } 66.3(1.9) \\
\text { Overweight }=57.7(4.6) \\
\text { At risk }=67.8(4.7) \\
\text { Normal weight= } 67.2(2.2) \\
\text { Underweight }=\text { not reported }\end{array}$ \\
\hline $\begin{array}{l}\text { Narksawat et al. } \\
\text { (2009) }\end{array}$ & Thailand & CS & 862 & $\begin{array}{l}12- \\
14\end{array}$ & $\begin{array}{l}\text { Quasi stratified } \\
\text { sampling of } 77 \text { districts }\end{array}$ & $3^{*}$ & 103 & $\begin{array}{l}\text { Prevalence DMFT } \\
=/>0\end{array}$ & $\begin{array}{l}\text { Thai Ministry of Public Health } \\
\text { manual used to classify children as } \\
\text { underweight, normal, overweight } \\
\text { and obese. } \\
\text { Inverse association between } \\
\text { nutritional status and DMFT }\end{array}$ & $\begin{array}{l}\text { Caries prevalence: } 62.1 \% \\
\text { Mn DMFT: } \\
\text { Overall sample = } 1.93(2.16) \\
\text { Normal weight: } 2.03(2.2) \\
\text { Underweight : } 2.19(2.19) \\
\text { Overweight: } 1.23(1.86) \\
\text { Obese: } 0.89(1.36) \\
\text { BMI: } \\
\text { Obese: } 6.3 \% \\
\text { Overweight: } 5.3 \% \\
\text { Normal : } 78.3 \% \\
\text { Underweight: } 10.1 \%\end{array}$ \\
\hline $\begin{array}{l}\text { Ngoenwiwatkul } \\
\text { \& Leela-Adisorn } \\
\text { (2009) }\end{array}$ & Thailand & CS & 212 & $6-7$ & Two primary schools & $\begin{array}{l}3- \\
\text { cavity } \\
\text { level }\end{array}$ & 103 & $\begin{array}{l}\text { Dmfs index Prevalence } \\
\text { dmfs }=/>0\end{array}$ & $\begin{array}{l}\text { Higher DMFT index with decreased } \\
\text { BMl } \\
\text { Gender (Boys) for dental caries } \\
\text { status (not dmfs) on primary } \\
\text { dentition }\end{array}$ & $\begin{array}{l}\text { Caries Prevalence: } 80.2 \% \\
\text { Mn dmfs =12.4 (12.3) } \\
\text { 70\% of children experienced } \\
\text { toothache } \\
\text { Mn BMI: } 15.5 \text { (4.6) } \\
\text { 45.8\% participants in low } \\
\text { percentile (5th<BMl-for- } \\
\text { age }<15 \text { th) }\end{array}$ \\
\hline $\begin{array}{l}\text { Olivira et al. } \\
\text { (2008) }\end{array}$ & Brazil & CS & 1018 & $1-5$ & $\begin{array}{l}\text { Randomly selected } \\
\text { from all children } \\
\text { attending for } \\
\text { vaccinations in } 17 \\
\text { Health centres in city } \\
\text { of Diadema }\end{array}$ & 4 & 84 & $\begin{array}{l}\text { dmsf index } \\
\text { Dichotomous dmfs } \\
=/>6\end{array}$ & $\begin{array}{l}\text { Mothers' and fathers' education } \\
\text { level, household overcrowding, and } \\
\text { number of children associated with } \\
\text { dental caries prevalence. } \\
\text { For ECC (LR): Caries prevalence } \\
\text { and mothers' education, age of } \\
\text { child, low BMI and clinically } \\
\text { detectable dental plaque. The final } \\
\text { hierarchical model demonstrated } \\
\text { that mothers' level of education, } \\
\text { age, dental plaque were associated } \\
\text { with and dental caries prevalence }\end{array}$ & $\begin{array}{l}\text { Caries Prevalence: } 23.4 \% \\
\quad S-E C C(d m f s \geq 6)=8.2 \%\end{array}$ \\
\hline
\end{tabular}


Table 3 Negative association between caries and BMI (higher caries associated with lower BMI) (Continued)

\begin{tabular}{|c|c|c|c|c|c|c|c|c|c|c|}
\hline & & & & & & & & & $\begin{array}{l}\text { For S-ECC (LR): BMI not } \\
\text { associated with severity } \\
\text { Mothers' education, family income } \\
\text {,age lower weight-for-age and } \\
\text { clinically detectable dental plaque } \\
\text { related to dental caries severity }\end{array}$ & \\
\hline $\begin{array}{l}\text { Sanchez-Perez } \\
\text { et al. (2010) }\end{array}$ & Mexico & L & $\begin{array}{l}110 \text { with } 88 \\
\text { at 4-year } \\
\text { follow up }\end{array}$ & $\begin{array}{l}7- \\
11\end{array}$ & $\begin{array}{l}\text { Public elementary } \\
\text { school in middle- } \\
\text { income area of Mexico } \\
\text { City }\end{array}$ & $\begin{array}{l}3- \\
\text { cavity } \\
\text { level }\end{array}$ & 57 & $\begin{array}{l}\mathrm{dmft} / \mathrm{DMFT} \mathrm{dmfs} / \\
\text { DMFS }\end{array}$ & $\begin{array}{l}\text { Higher dmfs scores } \\
\text { associated with } \\
\text { lower SE level }\end{array}$ & $\begin{array}{l}\text { Caries Prevalence: } \\
\text { At age } 7=59.1 \% \\
\text { At age } 8=55.7 \% \\
\text { At age } 9=59.1 \% \\
\text { At age } 10=50 \% \\
\text { At age } 11=40.9 \% \\
\text { BMl: Thin } 25 \% \\
\text { Normal } 45.5 \% \\
\text { At risk of overweight } 12.5 \% \\
\text { Overweight } 17 \%\end{array}$ \\
\hline $\begin{array}{l}\text { Sharma \& Hedge } \\
\text { (2009) }\end{array}$ & India & CS & $\begin{array}{l}500 \text { sample } \\
\text { of } \\
\text { convenience }\end{array}$ & $\begin{array}{l}8- \\
12\end{array}$ & $\begin{array}{l}\text { Department of } \\
\text { Pedodontics ad } \\
\text { Preventive Children } \\
\text { Dentistry, A.B Shetty } \\
\text { Memorial Institute of } \\
\text { Dental Sciences, } \\
\text { Mangalore }\end{array}$ & $\begin{array}{l}2 \text { - whether } \\
\text { initial } \\
\text { caries } \\
\text { was } \\
\text { included } \\
\text { is not } \\
\text { specified }\end{array}$ & 134 & DMFS/dmfs & $\begin{array}{l}\text { Higher rates of dental caries } \\
\text { (DMFS) in overweight and obese } \\
\text { children than normal weight } \\
\text { children. } \\
\text { Underweight children had } \\
\text { significantly higher DMFS rates } \\
\text { (but not dfs) than normal weight, } \\
\text { overweight and obese children. }\end{array}$ & $\begin{array}{l}\text { Mn DFMS: } \\
\text { Underweight: } 3.11 \\
\text { Normal weight: } 1.58 \\
\text { Overweight: } 2.48 \\
\text { Obese: } 2.85 \\
\text { Mn dfs: } \\
\text { Underweight: } 2.00 \\
\text { Normal weight: } 2.14 \\
\text { Overweight: } 4.79 \\
\text { Obese: } 3.25 \\
\text { BMl: } \\
\text { Underweight: } 8.6 \% \\
\text { Normal weight: } 58.4 \% \\
\text { Overweight/at risk: } \\
\text { 22.2\% } \\
\text { Obese: } 10.8 \%\end{array}$ \\
\hline
\end{tabular}


Table 4 No Association between dental caries and BMI

\begin{tabular}{|c|c|c|c|c|c|c|c|c|c|c|}
\hline Authors & Country & Design & $\mathbf{N}$ & Age & Sampling & $\begin{array}{l}\text { Dental } \\
\text { rating }\end{array}$ & $\mathrm{HDI}^{*}$ & Dental measure & $\begin{array}{l}\text { Significant cariogenic risk } \\
\text { factors }\end{array}$ & Sample demographics \\
\hline $\begin{array}{l}\text { Cereceda } \\
\text { et al. (2010) }\end{array}$ & Chile & CS & $\begin{array}{l}1190 \text { 'lower } \\
\text { middle class' } \\
\text { sample }\end{array}$ & $5-15$ & $\begin{array}{l}\text { Stratified random sampling } \\
\text { by gender and grade of } \\
\text { eight primary schools from } \\
\text { different districts of Santiago }\end{array}$ & $\begin{array}{l}3 \text { at cavity } \\
\text { level }\end{array}$ & 44 & COPD dmft & $\begin{array}{l}\text { No association between } \\
\text { caries and BMl }\end{array}$ & $\begin{array}{l}\text { Caries prevalence: } 79.5 \% \text {. } \\
\text { Caries prevalence by BMI: } \\
\text { Underweight: } 60 \% \\
\text { Normal weight: } 80 \% \\
\text { Overweight: } 78.1 \% \\
\text { Obese: } 79.9 \% \\
\text { BMI: } \\
\text { Underweight: } 1.2 \% \\
\text { Normal weight: } 51.6 \% \\
\text { Overweight: } 25 \% \\
\text { Obese: } 22 \%\end{array}$ \\
\hline Cinar et al. (2011) & Denmark & CS & 332 & 15 & $\begin{array}{l}\text { Eight Danish municipalities } \\
\text { selected for the purpose of } \\
\text { representing various } \\
\text { geographical areas of the } \\
\text { Denmark and various } \\
\text { degrees of urbanisation }\end{array}$ & $\begin{array}{l}\text { 2- cavity } \\
\text { level (for } \\
76 \% \text { of the } \\
\text { sample) } \\
\text { 3- cavity } \\
\text { level (for } \\
\text { the rest of } \\
\text { the sample) }\end{array}$ & 16 & DMFT & $\begin{array}{l}\text { No direct association } \\
\text { High loading on "health } \\
\text { cluster" for BMl, DMFT, } \\
\text { daily fruit consumption, } \\
\text { and non smoking }\end{array}$ & $\begin{array}{l}\text { Caries prevalence }=62 \% \\
\text { Mn DMFT: } 2.03(S D=3.01) \\
\text { BMI: } \\
\text { Mn BMI: } 21.30(S D=3.62) \\
\quad \text { Obese } 16 \%\end{array}$ \\
\hline $\begin{array}{l}\text { Cinar \& } \\
\text { Murtomaa } \\
\text { (2011) }\end{array}$ & Turkey & CS & $\begin{array}{l}611 \\
360 \text { public } \\
\text { school } \\
254 \text { private } \\
\text { school }\end{array}$ & $\begin{array}{l}10- \\
12\end{array}$ & $\begin{array}{l}\text { Two schools selected by } \\
\text { cluster sampling from high- } \\
\text { and low- socio-economic } \\
\text { level suburbs }\end{array}$ & $\begin{array}{l}3 \text { - cavity } \\
\text { level }\end{array}$ & 92 & DMFS & $\begin{array}{l}\text { Attendance at public school } \\
\text { associated with higher caries } \\
\text { rates and lower rates of BMI } \\
\text { DMFS, CPI and BMI shared } \\
\text { the "health" cluster among } \\
\text { both private and public school } \\
\text { children }\end{array}$ & $\begin{array}{l}\text { Caries prevalence: } \\
\text { Public School: } 91 \% \\
\text { Mn DMFS:4.44 (SD= 3.4) } \\
\text { Private School: } 70 \% \\
\text { Mn DMFS: } 2.64(S D=2.6) \\
\text { BMl: } \\
\text { Public School } \\
\text { Obese: } 25 \% ; \\
\text { Non-obese: } 75 \% \\
\text { Private School: } 70 \% \\
\text { Obese: } 40 \% ; \\
\text { Non-obese: } 60 \%\end{array}$ \\
\hline $\begin{array}{l}\text { Cinar \& } \\
\text { Murtomaa } \\
\text { (2008) }\end{array}$ & $\begin{array}{l}\text { Finland } \\
\text { and } \\
\text { Turkey }\end{array}$ & CS & $\begin{array}{l}949 \\
\text { Finnish } 338 \\
\text { Turkish } 611\end{array}$ & $\begin{array}{l}10 \\
-12\end{array}$ & $\begin{array}{l}\text { Matched suburbs. } \\
\text { Participating schools in } \\
\text { Turkey selected through } \\
\text { cluster sampling to } \\
\text { represent socio-economic } \\
\text { range of district. }\end{array}$ & $\begin{array}{l}\text { 1 Fin } \\
\text { 3Turk - } \\
\text { cavity level }\end{array}$ & $\begin{array}{l}22 \\
92\end{array}$ & $\mathrm{DMFT}$ & $\begin{array}{l}\text { No direct association found } \\
\text { between BMl and DMFT Turkish } \\
\text { children from public school had } \\
\text { lower mean BMl but higher Mn } \\
\text { DMFT than Turkish children in } \\
\text { private school Turkish sample } \\
\text { higher in BMl and dental caries } \\
\text { than Finnish sample. FA found } \\
\text { obesity and caries shared same } \\
\text { cluster. }\end{array}$ & $\begin{array}{l}\text { Caries Prevalence: } \\
\text { Finland: } 33 \% \\
\text { Turkey: } 84 \% \\
\text { Mn DMFT: } \\
\text { Finland: } 0.71(S D=1.54) \\
\text { Turkey } 2.93(S D=1.99) \\
\text { BMI } \\
\text { Finland: } 20 \% \text { obese } \\
\text { Turkey: } 28 \% \text { obese } \\
\text { Turkish private vs public } \\
\text { schools: } \\
\text { Caries prevalence: } \\
\quad \text { Public schools: } 92 \% ; \\
\text { Private: } 73 \%\end{array}$ \\
\hline
\end{tabular}


Table 4 No Association between dental caries and BMI (Continued)

\begin{tabular}{|c|c|c|c|c|c|c|c|c|c|c|}
\hline & & & & & & & & & & $\begin{array}{l}\text { Obesity: } \\
\quad \text { Public schools: } 39 \% \\
\text { Private: } 22 \%\end{array}$ \\
\hline $\begin{array}{l}\text { de Carvalho } \\
\text { Sales-Peres et al. } \\
\text { (2010) }\end{array}$ & Brazil & CS & 207 & 12 & $\begin{array}{l}\text { From eight schools (public } \\
\text { and private) in the Midwest } \\
\text { region of São Paulo }\end{array}$ & $\begin{array}{l}3 \text { - cavity } \\
\text { level }\end{array}$ & 84 & DMFT index & $\begin{array}{l}\text { No association between caries } \\
\text { and BMI Higher dental caries } \\
\text { was associated with lower } \\
\text { socioeconomic status }\end{array}$ & $\begin{array}{l}\text { Caries prevalence: } \\
\text { Private school: } 11.9 \% \\
\text { Public school: } 60.8 \% \\
\text { Mn Dmft: } \\
\text { Private schools: } 0.23 \\
\text { Public schools: } 2.16 \\
\text { BMI: } \\
\text { Private schools: } \\
\text { Low weight: } 35.59 \% \\
\text { Normal weight: } 55.93 \% \\
\text { Overweight: } 8.47 \% \\
\text { Obese: } 0 \% \\
\text { Public schools: } \\
\text { Low weight: } 41.22 \% \\
\text { Normal weight: } 52,03 \% \\
\text { Overweight: } 4.73 \% \\
\text { Obese: } 2.03 \%\end{array}$ \\
\hline $\begin{array}{l}\text { D'mello et al. } \\
\text { (2011) }\end{array}$ & $\begin{array}{l}\text { New } \\
\text { Zealand }\end{array}$ & CS & $\begin{array}{l}200 \text { sample } \\
\text { of } \\
\text { convenience }\end{array}$ & $3-8$ & $\begin{array}{l}\text { High caries of high anxiety } \\
\text { patient in the paediatric } \\
\text { dentistry clinics at the } \\
\text { University of Otago School } \\
\text { of Dentistry }\end{array}$ & 2 & 5 & $\begin{array}{l}\text { Dmft (number of } \\
\text { deciduous decayed, } \\
\text { missing and restored } \\
\text { teeth) }\end{array}$ & $\begin{array}{l}\text { No association between } \\
\text { BMl and caries }\end{array}$ & $\begin{array}{l}\text { Caries: } \\
\text { Mn Dmft = 6.1 ( SD =3.7) } \\
\text { Obese: } 24 \% \text { had dmft } \geq 8 \text {; } \\
\text { Overweight: } 37.5 \% \text { had } \\
\text { dmft } \geq 8 \\
35.4 \% \text { had dmft } \geq 8 \\
\text { BMl: } \\
\text { Obese: } 17 \%(8.5 \%) \\
\text { Overweight: } 23 \%(11.5 \%) \\
\text { Mn BMl = } 16.0 \%(2.0)\end{array}$ \\
\hline $\begin{array}{l}\text { Dye et al. } \\
\text { (2004) }\end{array}$ & USA & CS & 4236 & $2-5$ & NHANES III (1988-1994) & 2 & 4 & $\begin{array}{l}\text { DfsDichotomous: } \\
\text { dfs=/>0 Continuous } \\
0,1-2,3-5,>6 \\
\text { surfaces untreated }\end{array}$ & $\begin{array}{l}\text { No association between BMI } \\
\text { and caries Higher Caries } \\
\text { associated with: Low parental } \\
\text { education achievement, } \\
\text { Ethnicity (greater caries } \\
\text { experience in Mexican- } \\
\text { Americans than non-Hispanics) } \\
\text { Poverty status ( }=/<200 \% \text { of } \\
\text { the federal poverty level) Not } \\
\text { receiving breastmilk Not eating } \\
\text { breakfast daily Eating }<5 \\
\text { servings fruit \& veg Not having } \\
\text { dental visit within } 12 \text { months, } \\
\text { Age }\end{array}$ & $\begin{array}{l}\text { Caries Prevalence : } \\
2 \text { yrs: } 7.7 \% \\
3 \text { yrs: } 15.5 \% \\
4 \text { yrs: } 29.6 \% \\
5 \text { yrs: } 40.2 \% \\
\text { BMl: } \\
\text { Obese: } 23 \% \\
\text { Overweight: } 26.4 \% \\
\text { Normal \& Underweight: } \\
\text { 23.5\% }\end{array}$ \\
\hline
\end{tabular}


Table 4 No Association between dental caries and BMI (Continued)

\begin{tabular}{|c|c|c|c|c|c|c|c|c|c|c|}
\hline $\begin{array}{l}\text { Frisbee et al. } \\
\text { (2010) }\end{array}$ & USA & CS & 128 & 3-18 & 5 counties in West Virginia & 5 & 4 & $\begin{array}{l}\text { Parent report - Now } \\
\text { or ever had a cavity, } \\
\text { filling, tooth pulled } \\
\text { and overall dental } \\
\text { health }\end{array}$ & $\begin{array}{l}\text { No association between } \\
\text { BMl and caries }\end{array}$ & $\begin{array}{l}\text { Caries prevalence: } \\
\text { Now or ever had a cavity: } \\
61 \% \\
\text { Now or even had a } \\
\text { filling: } 56 \% \\
\text { Now or even had a tooth } \\
\text { pulled: } 36 \% \\
\text { Overall all dental health: } \\
\text { Excellent to very good: } 55 \% \\
\text { Poor to good: } 45 \% \\
\text { BMl: } \\
\text { Overweight or obese } 56 \%\end{array}$ \\
\hline $\begin{array}{l}\text { Granville- } \\
\text { Garcia et al. } \\
\text { (2008) }\end{array}$ & Brazil & CS & 2651 & $1-5$ & $\begin{array}{l}84 \text { private and public } \\
\text { elementary schools in } \\
\text { Recife (city) }\end{array}$ & $\begin{array}{l}\text { 3- cavity } \\
\text { level }\end{array}$ & 84 & $\mathrm{dmft}$ & $\begin{array}{l}\text { No association between } \\
\text { caries and BMI Significantly } \\
\text { higher decayed in public } \\
\text { school. }\end{array}$ & $\begin{array}{l}\text { Caries Prevalence 19\% } \\
\text { Public school: } 26.4 \% \\
\quad \text { Private school: } 11.4 \% \\
\text { dmft: 1.12 } \\
\text { BMl: } \\
\text { Obese: Overall 9\% } \\
\text { Public school: 4.6\% } \\
\text { Private school: } 13.6 \%\end{array}$ \\
\hline $\begin{array}{l}\text { Jamelli } \\
\text { et al. } \\
\text { (2010) }\end{array}$ & Brazil & $\begin{array}{l}\text { CS with } \\
\text { nested case } \\
\text { control }\end{array}$ & 689 & 12 & $\begin{array}{l}\text { Public school in the } \\
\text { municipality of Caruaru; low } \\
\text { socio-economic } 465 \text { cases } \\
\left(\text { DMFT >0); }{ }^{*} 182 \text { controls }\right. \\
(\mathrm{DMFT}=0){ }^{*} \text { no details on } \\
\text { matching criteria }\end{array}$ & No details & 84 & DMFT & $\begin{array}{l}\text { No association between } \\
\text { caries and BMICaries } \\
\text { associated with having } \\
\text { visited a clinician. }\end{array}$ & $\begin{array}{l}\text { Caries Prevalence: } 71.8 \% \\
\text { Mn DMFT = } 2.9 \\
\text { BMI: } \\
\text { Low weight: } 5.5 \% \\
\text { Risk of overweight: } 9.3 \% \\
\text { Overweigh/Obesity: } 3.2 \%\end{array}$ \\
\hline $\begin{array}{l}\text { Juarez-Lopez } \\
\text { \& Villa- } \\
\text { Ramos } \\
\text { (2010) }\end{array}$ & Mexico & CS & 189 & $3-6$ & $\begin{array}{l}\text { Convenience sample from } \\
\text { Iztapalapa's area of Mexico } \\
\text { City. }\end{array}$ & $\begin{array}{l}\text { Information } \\
\text { not } \\
\text { provided }\end{array}$ & 57 & dmf-t; dmf-s & $\begin{array}{l}\text { No association between } \\
\text { dental caries and weight } \\
\text { category (normal, } \\
\text { overweight and obese). } \\
\text { Gender (female) }\end{array}$ & $\begin{array}{l}\text { Caries prevalence: } \\
\text { Normal weight: } 77 \% \\
\text { Overweight: } 84 \% \\
\text { Obese: } 79 \% \\
\text { BMl: } \\
\text { Normal weight:33\% } \\
\text { Overweight: 33\% } \\
\text { Obese: 33\% }\end{array}$ \\
\hline $\begin{array}{l}\text { Jürgensen \& } \\
\text { Petersen } \\
\text { (2009) }\end{array}$ & Laos & CS & 621 & 12 & $\begin{array}{l}\text { Multistage random } \\
\text { sampling to select } 10 \\
\text { representative elementary } \\
\text { schools }\end{array}$ & $\begin{array}{l}3 \text { cavity } \\
\text { level }\end{array}$ & 138 & $\begin{array}{l}\text { Cavity level dmft/ } \\
\text { DMFT }\end{array}$ & $\begin{array}{l}\text { No association between } \\
\text { dental caries and BMl } \\
\text { Caries associated with } \\
\text { semi-urbanisation, poor } \\
\text { self-assessment of general } \\
\text { health, often experiencing } \\
\text { tooth ache in last } 12 \\
\text { months, and several time } \\
\text { being absent from } \\
\text { school in last } 12 \text { months, } \\
\text { higher economic status, } \\
\text { gender (girls), impairment of } \\
\text { quality of life (i.e., }\end{array}$ & $\begin{array}{l}\text { Caries Prevalence: } 56 \% \\
\text { DMFT = } 1.8(\mathrm{SE}=.09) \\
\mathrm{dmft}=.4(\mathrm{SE}=.04) \\
\text { BMI: } \\
\text { Normal weight: } 60 \% \\
\text { Overweight: } 8 \% \\
\text { Underweight: } 32 \%\end{array}$ \\
\hline
\end{tabular}




\begin{tabular}{|c|c|c|c|c|c|c|c|c|c|c|}
\hline $\begin{array}{l}\text { Kopycka- } \\
\text { Kedzierawski } \\
\text { et al. (2008) a }\end{array}$ & USA & CS & 10180 & $2-18$ & $\begin{array}{l}\text { NHANES III (1988-1994) } \\
\text { Nationally representative } \\
\text { sample }\end{array}$ & 2 & 4 & $\begin{array}{l}\text { DMFS and dfs } \\
\text { dichotomised as } \\
\text { either having caries } \\
\text { experience or not } \\
\text { Dfs and DFS in } \\
\text { children aged 2-11 } \\
\text { years were estimated }\end{array}$ & $\begin{array}{l}\text { Age 2-5 years: No association } \\
\text { between dental caries and } \\
\text { BMl Caries risk associated } \\
\text { with: poverty and } \\
\text { Mexican-American Ethnicity, } \\
\text { cotinine levels }\end{array}$ & $\begin{array}{l}\text { Caries prevalence (\%): } \\
\text { 2-5yrs old; Primary Caries } \\
\text { Overall:23.8 (1.4) } \\
\text { Overweight= } 23 \text { (3.6) } \\
\text { At risk = } 26.4(3) \\
\text { Normal weight= 23.5-(1.4) } \\
\text { 6-11yrs old; Primary Caries } \\
\text { Overall: } 49.5 \text { (1.6) } \\
\text { Overweight= 40.6 (4.7) } \\
\text { At risk = 45.5 (4.6) } \\
\text { Normal weight= } 51.4 \text { (1.6) } \\
\text { 6-11yrs old; Permanent Caries } \\
\text { Overall: } 25.9(1.7) \\
\text { Overweight= 17.6 (2.9) } \\
\text { At risk = } 29.9(4.0) \\
\text { Normal weight= 26.5 (1.7) } \\
\text { 12-18yrs; Permanent Caries } \\
\text { Overall: } 66.3(1.0) \\
\text { Overweight= 57.7 (4.6) } \\
\text { At risk =67.8 (4.7) } \\
\text { Normal weight= 67.2 (2.2) }\end{array}$ \\
\hline b & USA & CS & 7568 & $2-18$ & $\begin{array}{l}\text { NHANES 1999-2002 } \\
\text { Nationally representative } \\
\text { sample }\end{array}$ & 2 & 4 & $\begin{array}{l}\text { DMFS and dfs } \\
\text { dichotomised as } \\
\text { either having caries } \\
\text { experience or not } \\
\text { Dfs and DFS in } \\
\text { children aged 2-11 } \\
\text { years were estimated }\end{array}$ & $\begin{array}{l}\text { No association between } \\
\text { dental caries and BMl at } \\
\text { any age group } \\
\text { Age 2-5 years: Caries risk } \\
\text { associated with: Mexican- } \\
\text { American ethnicity, poverty, } \\
\text { time since the last dental } \\
\text { visit and blood lead levels } \\
\text { above median associated } \\
\text { with increased risk } \\
\text { 6-11 years of age: Caries risk } \\
\text { associated with: Mexican- } \\
\text { American ethnicity, time } \\
\text { since the last dental visit, } \\
\text { poverty and serum cotinine } \\
\text { levels }\end{array}$ & $\begin{array}{l}\text { Caries prevalence (\%): } \\
\text { 2-5yrs old: Primary Caries } \\
\text { Overall: } 28.2 \text { (1.8) } \\
\text { Overweight= 35.7 (5.8) } \\
\text { At risk = 24.3 (5.4) } \\
\text { Normal weight= 27.7 (1.8) } \\
\text { 6-11yrs old; Primary Caries } \\
\text { Overall: } 49 \text { (2.5) } \\
\text { Overweight= } 52.3 \text { (3) } \\
\text { At risk = 42.5 (4.1) } \\
\text { Normal weight= 49.7 (2.9) } \\
\text { 6-11yrs old; Permanent Caries } \\
\text { Overall: } 20.3(1.4) \\
\text { Overweight= } 23 \text { (2.4) } \\
\text { At risk = } 23.1 \text { (3.2) } \\
\text { Normal weight= 19.1 (1.7) } \\
\text { 12-18yrs; Permanent Caries } \\
\text { Overall: } 56.8(1.1)\end{array}$ \\
\hline
\end{tabular}

problems with eating, smiling

and sleeping), dental visits in

dental visits, preference for

intake vists, prefer dinks during

school hours and low atitude 
Table 4 No Association between dental caries and BMI (Continued)

\begin{tabular}{|c|c|c|c|c|c|c|c|c|c|c|}
\hline & & & & & & & & & $\begin{array}{l}12-18 \text { years of age: } \\
\text { Caries risk associated with: } \\
\text { Mexican- American ethnicity, } \\
\text { time since the last dental } \\
\text { visit, poverty and } \\
\text { serum cotinine levels }\end{array}$ & $\begin{array}{l}\text { Overweight= } 56.6(2.7) \\
\text { At risk }=58.2(2.9) \\
\text { Normal weight= } 56.6(1.2)\end{array}$ \\
\hline $\begin{array}{l}\text { Macek \& Mitola } \\
\text { (2006) }\end{array}$ & USA & CS & 7617 & $2-17$ & $\begin{array}{l}\text { NHANES } \\
1999-2002 \\
\text { Nationally } \\
\text { representative } \\
\text { sample }\end{array}$ & 2 & 4 & $\begin{array}{l}\text { Prevalence DMFT } \\
\text { /dmft }>0 \text { Severity geometric } \\
\text { mean for DMFT and } \\
\text { dmft }\end{array}$ & $\begin{array}{l}\text { No association between } \\
\text { dental caries prevalence } \\
\text { and weight categories } \\
\text { Dental caries severity } \\
\text { (geometric Mn DMFI) in } \\
\text { permanent dentition } \\
\text { associated with BMl: } \\
\text { overweight children had } \\
\text { lower geometric mean } \\
\text { DMFT }\end{array}$ & $\begin{array}{l}\text { Caries prevalence (\%): } \\
\text { 2-5 yrs (primary dentition) } \\
\text { Overall: } 27.5 \text { (1.7) } \\
\text { Underweight: } 18 \text { (5.2) } \\
\text { Overweight: } 36.1 \text { (6.4) } \\
\text { At risk of overweight: } \\
26.9 \text { (5.0) } \\
\text { Normal: } 28.1 \text { (.8) } \\
\text { 6-17 yrs (permanentdentition) } \\
\text { Overall: } 37.8(1.2) \\
\text { Underweight: } 31.7(6.3) \\
\text { Overweight: 38.8(1.7) } \\
\text { At risk of overweight: } \\
\text { 38.1 (2.3) } \\
\text { Normal: } 37.8(1.4) \\
\text { BMl: } \\
\text { Underweight: } 4 \% \\
\text { At risk o/weight: } 15 \% \\
\text { Overweight: } 15 \% \\
\text { Normal weight: } 63 \%\end{array}$ \\
\hline $\begin{array}{l}\text { Moreira et al. } \\
\text { (2006) }\end{array}$ & Brazil & CS & $\begin{array}{l}3330 \text { (1665 } \\
\text { obese; } 1665 \\
\text { normal-weight) }\end{array}$ & $\begin{array}{l}12- \\
15\end{array}$ & $\begin{array}{l}\text { Random } \\
\text { sampling from public } \\
\text { and private } \\
\text { schools in } \\
\text { Paralba }\end{array}$ & $\begin{array}{l}3 \text { cavity } \\
\text { level }\end{array}$ & 84 & DMFT & $\begin{array}{l}\text { No association between } \\
\text { dental caries and BMl } \\
\text { Higher rates of dental } \\
\text { caries associated in Public } \\
\text { versus Private school, age } \\
\text { ( } 12 \text { vs } 15 \text { yrs old) }\end{array}$ & $\begin{array}{l}\text { Caries Prevalence in: } \\
\text { Obese children: } 30 \% \\
\text { Private Schools: } 9.0 \% \\
\text { Public Schools: } 50.9 \% \\
\text { Normal weight: } 31 \% \\
\text { Private Schools: } 9.6 \% \\
\text { Public Schools: } 52.4 \% \\
\text { Mn DMFT in : } \\
\text { Obese children in } \\
\text { Private schools: } 1.90 \\
\text { Public schools: } 4.27 \\
\text { Normal-weight children in } \\
\text { Private schools: } 1.91 \\
\text { Public schools: } 4.25\end{array}$ \\
\hline $\begin{array}{l}\text { Pinto et al. } \\
\text { (2007) }\end{array}$ & USA & CS & $\begin{array}{l}135 \text { sample } \\
\text { of } \\
\text { convenience: } \\
81 \% \text { African } \\
\text { American }\end{array}$ & $\begin{array}{l}M= \\
8.7( \\
S D= \\
2.37)\end{array}$ & $\begin{array}{l}\text { Initial visit at } \\
\text { (urban) } \\
\text { Pennsylvania } \\
\text { Dental } \\
\text { School }\end{array}$ & 2 & 4 & $\mathrm{DS} / \mathrm{ds}$ & $\begin{array}{l}\text { No association between } \\
\mathrm{ds} / \mathrm{Ds} \text { and BMl or between } \\
\mathrm{ds} / \mathrm{Ds} \text { and gender or } \\
\text { ethnicity } \\
\text { Age significantly } \\
\text { associated with Ds/ds }\end{array}$ & $\begin{array}{l}\text { Mn Ds score } 2.06 \text { (Cl 1.4-2.7) } \\
\text { BMI: } \\
\text { Mn BMI } 18.36(3.5) \\
\text { At risk overweight: 12\% } \\
\text { Overweight: } 15 \%\end{array}$ \\
\hline
\end{tabular}


Table 4 No Association between dental caries and BMI (Continued)

\begin{tabular}{|c|c|c|c|c|c|c|c|c|c|c|}
\hline $\begin{array}{l}\text { Sadeghi et al. } \\
\text { (2011) }\end{array}$ & Iran & CS & 747 & $\begin{array}{l}12- \\
15\end{array}$ & $\begin{array}{l}\text { Twelve state } \\
\text { and private } \\
\text { secondary } \\
\text { schools }\end{array}$ & $\begin{array}{l}\text { 3- cavity } \\
\text { level }\end{array}$ & 88 & $\mathrm{DMFT}$ & $\begin{array}{l}\text { No association between } \\
\text { DMFT and BMI } \\
\text { Males had higher DMFT } \\
\text { than females }\end{array}$ & $\begin{array}{l}\text { Caries prevalence: } 83.9 \% \\
\text { Mn DMFT=2.83 (2.2) } \\
\text { Underweight: } 2.91 \text { (2.2) } \\
\text { Normal weight: } 2.92 \text { (2.3) } \\
\text { At risk for overweight: } \\
2.54 \text { (1.8) } \\
\text { Overweight: } 2.34 \text { (1.9) } \\
\text { BMl: } \\
\text { Underweight: } 7.5 \% \\
\text { Normal weight: } 72.8 \% \\
\text { At risk for overweight: 13.8\% } \\
\text { Overweight: 5.9\% }\end{array}$ \\
\hline $\begin{array}{l}\text { Scheutz et al. } \\
\text { (2007) }\end{array}$ & Tanzania & $\begin{array}{l}\text { L 1997, } \\
1999 \text { and } \\
2003\end{array}$ & 218 & $\begin{array}{l}\sim 6- \\
14\end{array}$ & $\begin{array}{l}\text { Two primary } \\
\text { schools } \\
\text { ('Affluent' } \\
\text { and 'Poor') in } \\
\text { Dar es Salaam }\end{array}$ & $\begin{array}{l}3 \text { cavity } \\
\text { level }\end{array}$ & 152 & DMFS & $\begin{array}{l}\text { No association between } \\
\text { DMFS and low BMl }\end{array}$ & $\begin{array}{l}\text { Caries: } \\
\text { DMFS at baseline: } \\
\text { Cohort } 1=0.33 \\
\text { Cohort } 2=0.37 \\
\text { Cohort } 3=0.32\end{array}$ \\
\hline $\begin{array}{l}\text { Sheller et al. } \\
\text { (2009) }\end{array}$ & USA & $\begin{array}{l}\text { Retrospective } \\
\text { case study }\end{array}$ & $\begin{array}{l}293 \text { children } \\
\text { with severe } \\
\text { early } \\
\text { childhood } \\
\text { caries }\end{array}$ & $2-5$ & $\begin{array}{l}\text { Thirty } \\
\text { different } \\
\text { state, } \\
\text { low income } \\
\text { population }\end{array}$ & 1 & 4 & $\begin{array}{l}\mathrm{dmft} \\
\text { Number teeth } \\
\text { with Pulp involvement }\end{array}$ & $\begin{array}{l}\text { No association between } \\
\text { dmft and BMl } \\
\text { Other factors associated } \\
\text { with higher dmft/pulp } \\
\text { involvement were Age } \\
\text { (older) and ethnicity } \\
\text { (Asian and 'not reported') }\end{array}$ & $\begin{array}{l}\text { Caries Prevalence: } 100 \% \\
\text { Mn dmft: =11.8 } \\
\text { Underweight: } 11.6 \text { (1.5) } \\
\text { Normal weight: } 11.9(.5) \\
\text { At risk for overweight: } \\
11.1 \text { (1.4) } \\
\text { Overweight: } 12.2 \text { (1.4) } \\
\text { Mn pulp involved teeth } \\
=4.1 \\
\text { Underweight: } 4.5 \text { (1.5) } \\
\text { Normal weight: } 4.0(.5) \\
\text { At risk for overweight: } \\
\text { 4.0 (1.5) } \\
\text { Overweight: } 3.9 \text { (1.0) } \\
\text { BMl: } \\
\text { Underweight: 12\% } \\
\text { Normal weight: 69\% } \\
\text { At risk for overweight: 9\% } \\
\text { Overweight: } 11 \%\end{array}$ \\
\hline $\begin{array}{l}\text { Tramini et al. } \\
(2009)^{1}\end{array}$ & France & CS & 835 & 12 & $\begin{array}{l}\text { Randomly } \\
\text { selected from } \\
\text { Montpellier } \\
\text { schools }\end{array}$ & $\begin{array}{l}3 \text { caries in } \\
\text { to dentine }\end{array}$ & 20 & $\mathrm{DMFT}$ & $\begin{array}{l}\text { No association between } \\
\text { DMFT and BMl } \\
\text { Dental caries associated } \\
\text { with higher sugar } \\
\text { consumption, } \\
\text { soft drink consumption } \\
\text { and gender }\end{array}$ & $\begin{array}{c}\text { Caries prevalence: } 51.7 \% \\
\text { Underweight }=40 \% \\
\text { Normal weight }=51.7 \% \\
\text { Overweight }=50.5 \% \\
\text { Obese }=62.5 \% \\
\text { Mn DMFT: } 1.47 \\
\text { Underweight }=0.73 \\
\text { Normal weight }=1.47 \\
\text { Overweight }=1.58 \\
\text { Obese }=1.66 \\
\text { Mn BMI }=18.9\end{array}$ \\
\hline
\end{tabular}


Table 4 No Association between dental caries and BMI (Continued)

\begin{tabular}{|c|c|c|c|c|c|c|c|c|c|c|}
\hline $\begin{array}{l}\text { Tripathi et al. } \\
\text { (2010) }\end{array}$ & India & CS & 2688 & $6-17$ & $\begin{array}{l}\text { Selected from a private and } \\
\text { two governments schools } \\
\text { in Bareilly }\end{array}$ & $\begin{array}{l}3 \text { cavity } \\
\text { level }\end{array}$ & 134 & DMFT & $\begin{array}{l}\text { No association between } \\
\text { DMFT caries and weight } \\
\text { category. }\end{array}$ & $\begin{array}{l}\text { Caries prevalence = 19.1\% } \\
\text { Private schools= } 27.6 \% \\
\text { Govemment schools = 9.6\% } \\
\text { BMl: Obese: } 4.7 \% \\
\text { Private schools: } \\
\text { Obese } 75 \% ; \\
\text { Non-obese } 926 \% \\
\text { Govemment schools: } \\
\text { Obese } 1.57 \% ; \\
\text { Non-obese } 98.4 \%\end{array}$ \\
\hline $\begin{array}{l}\text { Van Gemert- } \\
\text { Schriks et al. (2011) }\end{array}$ & Suriname & CS & 380 & 6 & $\begin{array}{l}\text { Seventeen schools from } 2 \\
\text { different regions of the } \\
\text { Rainforst, selected from the } \\
\text { databases of the Medical } \\
\text { Mission }\end{array}$ & 3 & 104 & $\begin{array}{l}\text { Total caries experience } \\
\text { (dmfs) Total-ds } \\
\text { Dichotomised dentogenic } \\
\text { infections }>0 /=0\end{array}$ & $\begin{array}{l}\text { No association between } \\
\text { dmfs and BMI } \\
\text { Higher rates of caries } \\
\text { associated with reduced } \\
\text { height suggesting caries } \\
\text { is impacting on normal } \\
\text { growth and development. }\end{array}$ & $\begin{array}{l}\text { Caries: } \\
\quad \text { M total-ds: } 14.0(+/-10.1)\end{array}$ \\
\hline
\end{tabular}

${ }^{1}$ Authors found a negative association using a logistic and Poisson regression models but report no association after undertaking a zero-inflated and zero-inflated negative binomial regression models. $\mathrm{HDI}^{*}=$ Human Development Index. 
Table 5 Comparison of sample demographics and method of dental examination for studies finding different associations between body mass index and dental caries

\begin{tabular}{|c|c|c|c|}
\hline & Positive association & Negative association & No association \\
\hline Sample & 17 & 9 & 24 \\
\hline \multirow[t]{2}{*}{ Median Human Development Index } & $10($ Mean = 21.9; & $84($ Mean = 72.11; & $50.50($ Mean = 55.33; \\
\hline & $S D=32.9)$ & $S D=45.55)$ & $S D=49.69)$ \\
\hline \% Rank 1 to 2 dental examination & 70.6 & 33.3 & 45.8 \\
\hline \multirow[t]{2}{*}{ Median caries prevalence $\%$} & $48.05($ Mean $=51.24$ & $59.1($ Mean = 58.03; & $50.35($ Mean $=49.47$ \\
\hline & $S D=23.39)$ & $S D=21.59)$ & $S D=27.21)$ \\
\hline \multirow[t]{2}{*}{ Median dmft/DMFT } & $2.12($ Mean = 2.13; & $3.11($ Mean = 4.69; & $2.04($ Mean = 3.04; \\
\hline & $S D=1.21)$ & $S D=3.41)$ & $S D=3.40)$ \\
\hline \multirow[t]{2}{*}{ Median \% sample overweight } & $21.65($ Mean = 26.15; & $20.55($ Mean = 18.77; & $20($ Mean = 24.65; \\
\hline & $S D=14.87)$ & $S D=15.10)$ & $S D=17.55)$ \\
\hline \multirow[t]{2}{*}{ Median \% sample underweight } & $4.2($ Mean = 12.90; & $25($ Mean $=23.32 ;$ & $12($ Mean = 18.75; \\
\hline & $S D=24.56)$ & $S D=15.11)$ & $S D=14.48)$ \\
\hline
\end{tabular}

that any underweight children were absorbed within the 'normal-weight' category for comparison with children at-risk and overweight because no information was provided about their exclusion. If underweight children have systematically higher rates of caries than normalweight children, and there is evidence that this may be the case (for example, [9]), caries incidence and severity in the normal-weight group would be inflated by the inclusion of underweight children, and potential differences between normal-weight and overweightgroups attenuated. The non-significant trends found in the NHANES studies $[19,25,26]$ might be explained in this manner. Underweight children should be excluded from the analysis if insufficient in number to instantiate a comparison group, and it would be helpful if this information was provided.

\section{Dental caries associated with higher BMI}

Table 2 contains a summary of the 17 studies that found that dental caries prevalence or severity is higher in children and adolescents with higher BMI/body fat index. Caries prevalence ranged from $17.9 \%$ [27] to 90\% [23] but the population-based studies reported prevalence rates between $42 \%$ [25] and 67\% [5]. The dmft/DMFT rates were positively skewed, ranging from 1.4 [28] to 6.9 [23] with most studies reporting dmft/DMFT of approximately 2.0. The BMI distributions in this group appeared to be negatively skewed with seven studies $[11,16,21,25,28-30]$ reporting that approximately 2 to $10 \%$ of their samples were underweight and 20 to $30 \%$ of their samples were overweight or obese. Six studies [23,24,27,31-33] appeared to have no underweight children in their samples, and remaining studies failed to provide sufficient detail $[14,34,35]$. The samples in these studies therefore appeared to be positively skewed for dental caries and negatively skewed for BMI.

\section{Other factors associated with caries}

Overall, the findings from this group of studies suggest that dental caries, as measured by prevalence or severity in deciduous and permanent teeth, increases with increased BMI. There is some suggestion that this association is moderated by age; three studies [25,28,31] found the association between high BMI and high dental caries appeared at around the age of 5 to 9 years and not earlier, although two [14, 25,] found the association in children under age 5 years. Other factors found to influence caries prevalence and severity rates include consuming 'caries risk products' more than three times per day at age 1 year [5]; sweets more than once per week at age 3 years [5]; higher rates of soda pop consumption [29,34]; sugar consumption [27]; lower socioeconomic status/poverty index/living in a disadvantaged neighborhood [24,25,29,34]; ethnicity [5], specifically being Hispanic or non-Hispanic black [25]; dental fatalism in caregivers [34]; parents' poor attitudes to dental health and psychosocial factors [5]; less educated parent [29]; and heavier mothers [29].

\section{Dental caries associated with lower BMI}

Table 3 summarizes the nine studies that found a negative association between dental caries and BMI. Caries prevalence ranged from $23.4 \%$ [36] to approximately $80 \%[37,38]$. The $\mathrm{dmft} / \mathrm{DMFT}$ rates ranged from 2.07 [16] to 14.0 [36]. Two studies reported odontogenic infections in more than half of the samples $[37,38]$. Three of the nine studies in Table 3 described the distribution of both underweight and overweight children in their sample $[9,17,21]$.

\section{Other factors associated with caries}

Other factors associated with dental caries were social deprivation/affluence/lower socioeconomic level/lower household education level $[9,19,39,40]$, all of which have also been associated with obesity [41], and larger families [36,37]. 


\section{No association between dental caries and BMI}

Table 4 summarises the 23 studies that found no association between dental caries and BMI. Caries prevalence ranged from $19.1 \%$ [19] to $91 \%$ [42] with higher prevalence in public schools (60.8\% [43], 26.4\% [44], 52.4\% [45]) compared to private schools (11.9\% [43], 11.4\% [44], 9.6\% [45]). Mean dmft/DMFT varied from .23 in private school children in Brazil [43] to 14 in children from remote areas of the rain forest of Suriname [46], with most studies reporting approximately 2.0. Seven of the 22 studies provided a breakdown of BMI categories, and the distributions included both a negative $[26,47-49]$ and a positive skew $[43,50,51]$. Unfortunately, many studies described only the proportion of obese and nonobese participants. Failure to distinguish between normal-weight and underweight children makes it difficult to know how well the association between caries and BMI was tested in underweight children or between normal-weight and overweight chidlren. Proportions of obese children reported in these studies ranged from 20 to $50 \%[8,15,20,25,44,48,52]$.

\section{Other factors associated with caries}

Factors that were found to be associated with caries were ethnicity $[8,47]$, low parental education achievement $[8,19]$, poverty/lower socioeconomic level (below the federal poverty level $[8,19,43])$, higher SES level [50], female gender [48,53] not eating breakfast daily [8], eating fewer than five servings of fruit and vegetables daily [8], attending public school [20,42,44,50], school absenteeism [50], soft drink consumption [50,54], low health attitude [50], and reduced height [46].

\section{Emergent patterns}

Table 5 summarizes emergent patterns that may help account for the disparate findings between the three sets of studies. These patterns primarily relate to differences in the method of dental examination and sample demographics. These differences are noted below.

1. Method of dental examination: Studies that found a positive association between BMI and dental caries primarily used dental examinations that permitted the detection of initial caries (that is, ranked 1 or 2) whereas studies finding a negative, or no association, tended to use field examination methods (that is, ranked 3 or below), which underestimated caries rates (see Tables 2, 3 and 4 for detail and Table 5 for summary). A chi-square test of independence revealed this association approached significance $\left(\chi^{2}(2)=5.19 ; P=.07\right)$.

2. Sample differences: Studies that found a positive association between BMI and dental caries were primarily conducted in Europe and the United States (see Table 2); those that found an inverse association were primarily conducted in Asia and South America (see Table 3); and those that found no association were largely conducted in the United States, South America and Europe. In an effort to quantify and compare the level of development of the countries represented in the three sets of studies, the Human Development Index (HDI) ranking for each sample is provided in Tables 2, 3 and 4. The HDI is a composite human development index that combines life expectancy, educational attainment and income to rank and compare the level of development of different countries [53]. A Kruskal-Wallis test revealed a significant difference in HDI between the three sets of studies $\left(\chi^{2}(2)=7.067, P<.05\right)$ Those studies finding a positive association between dental caries and BMI used samples from more highly developed countries than studies that found an inverse association $(U=27.00, P<.01)$.

3. Prevalence: Dental caries prevalence tended to be similar across the three sets of studies although it should be recognized that studies summarized in Tables 3 and 4 tended to exclude non-cavitated lesions and therefore underestimate caries prevalence. Caries severity, as measured by $\mathrm{dmft} /$ DMFT tended to be higher in studies finding an inverse association between BMI and dental caries than studies finding a positive or no association. A Kruskal-Wallis analysis found a significant difference $\left(\chi^{2}(2)=7.255, P<.05\right)$. Post hoc tests, using Mann Whitney $\mathrm{U}$, suggests studies that found an inverse association between dental caries and BMI had involved samples with a significantly higher $\mathrm{dmft} /$ DMFT rate than studies finding no association $(U=67, P=0.46)$ or a positive association $(U=73.50$, $P=0.03)$.

4. BMI distribution: The nature of the distribution of BMI differed across the three sets of studies, which is problematic. In order to determine whether BMI is adequately tested against dental caries it is important to ensure that the sample represents the full range of BMI categories (that is, low (underweight), normal, and high (overweight/obese)). Only 68\% of the studies reported the proportion of their sample that was overweight or obese, and $48 \%$ reported the proportion that was underweight. A KruskalWallis test found underweight participants were significantly under-represented in studies that found a positive association between BMI and dental caries $\left(\chi^{2}(2)=6.877, P<.05\right)$ compared with studies finding an inverse associations $(U=8, P<.05)$, and no association $(U=23, P=.046)$. No differences were found between studies in the proportion of the sample that was overweight or obese although only 
half of studies that found negative association reported the proportion of the sample that was overweight or obese.

A related issue is the inappropriate collapsing of groups across BMI categories as mentioned earlier. Studies that collapse across BMI categories assume a linear relationship exists between dental caries and BMI. If the relationship is non-linear, collapsing across BMI groups can attenuate any between-group differences between overweight or underweight children and the normal-weight reference group. Similarly, analyses that assume a linear relationship between BMI and dental caries, such as bivariate correlations may not detect a non-linear relationship. Given the evidence that dental caries is associated with both high and low BMI, analyses appropriate for non-linear associations might be more appropriate. Only four of the 23 studies that found no association between dental caries and BMI compared dental caries across low-weight, normalweight, and over-weight groups [26,48-50]. Of the remaining 20 studies, five studies used analyses that assumed a linear relationship [43,46,47,52,55], and 11 studies compared overweight or obese children with 'non-obese' or 'normal-weight' children [8,15,19,22,43$45,51,54,56]$ without providing information about how they accounted for underweight children in their sample. Three studies excluded underweight children to permit a comparison between obese and normal-weight children $[20,42,57]$.

\section{Discussion}

The results of this systematic review show that there is still significant disagreement as to the existence and nature of an association between dental caries and BMI. Forty-eight percent of studies reviewed found no association between dental caries and BMI; 35\% found a positive association and 19\% found an inverse association. Our results are therefore consistent with those of Kantovitz et al. [10]; however, we would like to speculate further about the association between dental caries and BMI, offer possible reasons for the disparity in findings, and make recommendations for modifications to future research in this area. We suggest that dental caries and BMI are related in a nonlinear fashion with more dental caries occurring in individuals with either higher or lower BMI. Furthermore we suggest that methodological factors including sample demographics, the sensitivity of the dental examination, and the nature of the data analyses undertaken influence whether or not the association is detected.

The evidence supporting an inverse relationship between dental caries and BMI comes from studies in developing countries and/or from samples with severe dental caries. Severe dental caries may well reduce eating ability thereby resulting in poor weight gain. Evidence to support this can be seen when, postcomprehensive dental rehabilitation, young children with early childhood caries show significantly increased growth velocities compared with controls [58]. Malnutrition could also predispose to dental caries; deficiencies in protein or energy foods may lead to protein-energy malnutrition, decreased salivary flow, calculus formation, high levels of caries and reduced growth [42]. Chronic malnutrition, particularly during the early years, has been shown to increase susceptibility to dental caries in the primary dentition (for example, [59]) perhaps via enamel hypoplasia and salivary hypofunction [60]. Alternatively both outcomes could be influenced by a third variable (or cluster of variables) such as those associated with SES.

Socioeconomic factors clearly impact the development of caries and need to be understood. Lower socioeconomic status (SES), whether measured by living in a disadvantaged neighborhood, below the poverty line, attending public versus private school (for example $[24,25,29])$, is associated with higher caries indices. A cluster of factors such as low parental education level, ethnicity, limited access to services and support, associated with low SES, are also associated with higher caries rates (for example, [25,29,34]). However dental caries is not limited to those from low SES backgrounds; children from high SES groups, whose parents have high expendable income, can have increased exposure to fermentable carbohydrates and may be at an increased risk of dental caries (for example, [50,61]). Obesity is also experienced in children from higher SES backgrounds [2,7].

The studies that support a positive association between BMI and dental caries include those in which 1) samples were negatively skewed for BMI with underweight children underrepresented; 2) samples were from highly developed countries with high standards of living and improved access to public health (presumably including fluoride); and 3) studies that tended to use more sensitive dental examinations that permitted the detection of initial caries. It is possible that the development of dental caries in more affluent populations follows a different pathway to that in less-affluent populations. Dental caries is likely to be slower progressing (for example due to increased fluoride exposure or reduced likelihood of chronic malnutrition) and it may therefore be critical to include initial caries in caries diagnosis in more affluent populations in order to detect subtle differences in dental health.

It is not surprising to find that children who are overweight or obese also have relatively high levels of dental caries given that overweight children tend to consume high levels of soda [62] and other energy-dense foods 
[63], many of which are cariogenic and obesogenic. Modeer et al. [32] suggest that obese children are at risk of dental caries because they have reduced salivary flow, something also found in underweight children [60], and which is associated with protein-deficient malnutrition. Obese children may well suffer from protein deficient malnutrition if their energy intake is made up of high carbohydrate, highly processed foods.

Almost half of the studies included in this review found no association between dental caries and BMI. We have suggested that several methodological factors may have contributed to these findings including: 1) a failure to include initial caries in caries assessment, which is important given the possibility that the ratio of initial to manifest caries may be different in overweight and underweight samples; 2) a failure to appropriately sample from the full range of BMI scores to adequately represent underweight, normal, and overweight and obese participants; 3) the undertaking of analyses that assume a linear relationship; and 4) the collapsing across BMI categories when making between-group comparisons (for example [64]). Costacurta et al. [16] suggest that BMI may not be the best measure of body fat composition when testing the association between dental caries and obesity. They suggest that misclassification of childhood obesity using BMI might account for the failure of studies to detect the association between child adiposity and dental caries (in samples where children are normal-weight and overweight).

Based on our findings in this review we can offer several recommendations for future research in this field:

1. When screening for dental caries, initial caries should be included in the caries measure, and the same diagnostic criteria should be used by all researchers to enable comparisons to be made across studies. Despite attempts to standardize caries assessment for research purposes since 2002 (for example, ICDAS, 2009 [65]) considerable disparity in diagnostic criteria still exists.

2. To permit some level of comparison to be made across studies, it would be useful to have access to details about sample parameters for target variables such as BMI and caries parameters, prevalence rates, a breakdown of BMI groups and demographic information such as SES.

3. The possibility of a non-linear association between dental caries and BMI should be considered, and appropriate analyses performed when testing the relationship.

4. Prospective longitudinal studies are needed to explore the causal relationships between the variables alluded to in this systematic review and to inform interventions. Longitudinal studies are also required to study the long-term association between dental health indices and broader general health outcomes because dental indices may provide a more reliable indicator of future health outcomes than BMI. BMI fluctuates throughout life and may not, at any given point in time, provide an accurate representation of a lifetime of dietary and health behaviors. Also, it is important to establish first, the time required for the association between obesity and caries to manifest, and second, to determine whether the association changes over time.

5. Given the impact of parent factors such as socioeconomic status, education level, diet, dental fatalism, health attitudes and so on, on the development of dental caries as found across studies, a focus on familial or parental influences is warranted. Such an approach might help explicate the effect of wider ecological influences, such as SES on the development of dental caries, especially in early childhood when dietary habits are being formed and implemented by parents.

\section{Conclusions}

There is evidence that dental caries is associated with both high and low BMI. Although the precise nature of these associations remains unclear, it is possible that different factors are involved in the development of caries in children with high and low BMI and in high and low socio-economic strata. Evidence supports the proposal of combined strategies to target both dental caries and obesity simultaneously, however further investigation of the association between the diseases and among their predictors is required. Specific attention should be given to longitudinal studies to gauge the association between early childhood caries and health outcomes in adolescence and adulthood, to the inclusion of younger children (aged 0 to 6 years) in the samples, to the perseverance of dietary and health-related behaviors developed during the preschool years, and to parental or familial influences on the development of these patterns. Abbreviations: BMI, body mass index; DMFT/dmft, decayed (D/d ), missing $(\mathrm{M} / \mathrm{m})$, filled $(\mathrm{F} / \mathrm{f})$ surfaces $(\mathrm{S} / \mathrm{s})$ or teeth $(\mathrm{T} / \mathrm{t})$ index in the permanent/primary dentition; HDI, human development index; NHANES, National Health and Nutrition Examination Survey; SES, socioeconomic status.

\section{Additional files}

Additional file 1: Review of included studies' methodology.

Additional file 2: Excluded papers with reasons.

\section{Competing interests}

The authors have no competing interests to declare. 


\section{Authors' contributions}

$\mathrm{CB}$ carried out the initial database searches and preliminary readings. $\mathrm{MH}$ reread abstracts and articles for final selection and prepared the draft. All authors read and approved the final manuscript.

\section{Acknowledgements}

The authors would like to acknowledge the valuable feedback received on early drafts of this manuscript by Francisco Vázquez-Nava, PhD, and Julie Eichenberger Gilmore, PhD.

\section{Author details}

${ }^{1}$ School of Psychology, Deakin University, 221 Burwood Highway, Burwood, Melbourne, Victoria 3125, Australia. ${ }^{2}$ Melbourne Dental School,Faculty of Medicine Dentistry and Health Sciences, The University of Melbourne, 720 Swanston Street, Melbourne 3010, Australia. ${ }^{3}$ Department of Paediatrics, Faculty of Medicine, Dentistry and Health Sciences, The University of Melbourne, 720 Swanston Street, Melbourne 3010, Australia. ${ }^{4}$ Murdoch Children's Research Institute, Royal Children's Hospital, Flemington Road, Parkville, Victoria 3052, Australia.

Received: 18 January 2012 Accepted: 9 October 2012 Published: 21 November 2012

\section{References}

1. National Institute of Dental and Craniofacial Research-National Institute of Health: Oral health in America: A report of the surgeon General (Executive summary). http://www.nidcr.nih.gov/DataStatistics/SurgeonGeneral/Report/ ExecutiveSummary.htm.

2. Wake M, Nicholson JM, Hardy P, Smith K: Preschooler obesity and parenting styles of mothers and fathers: Australian national Population study. Pediatrics 2007, 120:e1520-e1527.

3. Access Economics: The Growing Cost of Obesity in 2008: three years on. http:// diabetesaustralia.com.au/PageFiles/7830/FULLREPORTGrowingCostOfObesity2008.pdf.

4. Australian Institute of Health and Welfare: Australia's health 2010. Australians health series no. 12. Canberra: AlHW; 2010. Cat. No AUS 122.

5. Alm A, Fåhraeus C, Wendt LK, Koch G, Andersson-Gäre B, Birkhed D: Body adiposity status in teenagers and snacking habits in early childhood in relation to approximal caries at 15 years of age. Int J Paediatr Dent 2008, 18:189-196.

6. Armfield JM, Roberts-Thomson KF, Spencer AJ, Dental Statistics and Research Series: The Child Dental Health Survey, Australian 1999: Trends across the 1990s. Cat. no. DEN 95: AlHW; 2003.

7. Booth M, Dobbins T, Okely A, Denney-Wilson E, Hardy L: Trends in the prevalence of overweight and obesity among young Australians, 1985-1997, and 2004. Obesity 2007, 15:1089-1095.

8. Dye BA, Shenkin JD, Ogden CL, Marshall TA, Levy SM, Kanellis MJ: The relationship between healthful eating practices and dental caries in children aged 2-5 years in the United States, 1988-1994. J Amer Dent Assoc 2004, 135:55-66.

9. Sanchez-Perez L, Irigoyen M, Zepeda M: Dental caries, tooth eruption timing and obesity: a longitudinal study in a group of Mexican schoolchildren. Acta Odontol Scand 2010, 68:57-64.

10. Kantovitz KR, Pascon FM, Rontani RMP, Gavião MBD: Obesity and denta caries-A systematic review. Oral Health Prev Dentistry 2006, 4:137-144.

11. Willershausen B, Haas G, Krummenauer F, Hohenfellner K: Relationship betweem high weight and caries frequency in German elementary school children. Eur J Med Res 2004, 9:400-404.

12. Chen W, Chen P, Chen SC, Shih WT, Hu HC: Lack of association between obesity and dental caries in three-year-old children. Acta Paed Sin 1998, 39:109-111.

13. Tuomi T: Pilot study on obesity in caries prediction. Comm Dental Oral Epid 1989, 17:289-291.

14. Reifsnider E, Mobley C, Mendez D: Childhood obesity and Early Childhood Caries in a WIC Population. J Multicult Nurs Health 2004, 10:24-31.

15. Frisbee SJ, Chambers CB, Frisbee JC, Goodwill AG, Crout RJ: Self-reported dental hygiene, obesity, and systemic inflammation in a pediatric rural community cohort. BMC Oral Health 2010, 10:21.

16. Costacurta M, Di Renzo L, Bianchi A, Fabiocchi F, De Lorenzo A, Docimo R: Obesity and dental caries in paediatric patients. A cross-sectional study. Eur J Paediatr Dent 2011, 12:112-116.
17. Narksawat K, Tonmukayakul U, Boonthum A: Association between nutritional status and dental caries in permanent dentition among primary schoolchildren aged 12-14 years. Thailand. Southeast Asian J Trop Med Public Health 2009, 40:338-344.

18. Alm A: On dental caries and caries-related factors in children and teenagers. Swed Dent J 2008, 195(Supp):7-63.

19. Kopycka-Kedzierawski DT, Auinger P, Billings RJ, Weitzman M: Caries status and overweight in 2- to 18-year-old US children: findings from national surveys. Community Dent Oral Epidemiol 2008, 36:157-167.

20. Cinar B, Murtomaa $\mathrm{H}$ : Clustering of obesity and dental health with lifestyle factors among Turkish and Finnish pre-adolescents. Obes Facts 2008, 1:196-202.

21. Sharma A, Hegde AM: Relationship between body mass index, caries experience and dietary preferences in children. J Clin Pediatr Dent 2009, 34:49-52.

22. D'Mello G, Chia L, Hamilton SD, Thomson WM, Drummon BK: Childhood obesity and dental caries among paediatric dental clinic attenders. Int $J$ Paediatr Dent 2011, 21:217-222.

23. Bailleul-Forestier I, Lopes K, Souames M, Azoguy-Levy S, Frelut ML, Boy-Lefevre ML: Caries experience in a severely obese adolescent population. Int J Paediatr Dent 2007, 17:358-363.

24. Gerdin EW, Angbratt M, Aronsson K, Eriksson E, Johansson I: Dental caries and body mass index by socio-economic status in Swedish children. Community Dent Oral Epidemiol 2008, 36:459-465.

25. Hong L, Ahmed A, McCunniff M, Overman P, Mathew M: Obesity and dental caries in children aged 2-6 years in the United States: National health and nutrition examination survey 1999-2002. J Public Health Dent 2008, 68:227-233.

26. Macek MD, Mitola DJ: Exploring the association between overweight and dental caries among US children. Pediatr Dentistry 2006, 28:375-380.

27. Vázquez-Nava F, Vazquez-Rodriguez EM, Saldivar-Gonzalez AH, Lin-Ochoa D, Martinez-Perales GM, Joffre-Velazquez VM: Association between obesity and dental caries in a group of preschool children in Mexico. J Public Health Dent 2010, 70:124-130.

28. Willershausen B, Moschos D, Azrak B, Blettner M: Correlation between oral health and body mass index (BMI) in 2071 primary school pupils. Eur J Med Res 2007, 12:295-299.

29. Marshall TA, Eichenberger-Gilmore JM, Broffitt BA, Warren JJ, Levy SM: Dental caries and childhood obesity: roles of diet and socioeconomic status. Community Dent Oral Epidemiol 2007, 35:449-458.

30. Willerhausen B, Blettner M, Kasaj A, Hohenfellner K: Association between body mass index and dental health in 1,290 children of elementary schools in a German city. Clin Oral Investig 2007, 11:195-200.

31. Alm A, Isaksson H, Fahraeus C, Koch G, Andersson-Gare B, Nilsson M, Birkhed D, Wendt LK: BMI status in Swedish children and young adults in relation to caries prevalence. Swed Dent J 2011, 35:1-8.

32. Modéer T, Blomberg CC, Wondimu B, Julihn A, Marcus C: Association between obesity, flow rate of whole saliva, and dental caries in adolescents. Obes 2010, 18:2367-2373.

33. Martínez-Sotolongo B, Martínez-Brito I: Behavior of dental caries in 8 to 13 years-old obese and normal-weight students. Rev Med Electrón 2010 32:1684-1824

34. Ismail Al, Sohn W, Lim S, Willem JM: Predictors of dental caries progression in primary teeth. J Dent Res 2009, 88:270-275.

35. Hilgers KK, Kinane DE, Scheetz JP: Association between childhood obesity and smooth-surface caries in posterior teeth: a preliminary study. Paediatr Dent 2006, 28:23-28.

36. Oliveira LB, Sheiham A, Bonecker M: Exploring the association of dental caries with social factors and nutritional status in Brazilian preschool children. Eur J Oral Sci 2008, 116:37-43.

37. Benzian $H$, Monse $B$, Heinrich-Weltzien $R$, Hobdell M, Mulder J, van Palenstein Helderman W: Untreated severe dental decay: A neglected determinant of low Body Mass Index in 12-year-old Filipino children. BMC Public Health 2011, 11:558

38. Ngoenwiwatkul Y Leela-Adisorn N. Effects of dental caries on nutritional status among first-grade primary school children. Asia Pac J Public Health 2009, 21:177-183.

39. Cameron FL, Weaver LT, Wright CM, Welbury RR: Dietary and social characteristics of children with severe tooth decay. Scott Med J 2006, $51: 26-29$ 
40. Floyd B: Associations between height, body mass, and frequency of decayed, extracted, and filled deciduous teeth among two cohorts of Taiwanese first graders. Am J Phys Anthropol 2009, 140:113-119.

41. MCLaren L: Socioeconomic status and obesity. Epidemiol Rev 2007, 29:29-48.

42. Cinar AB, Murtomaa $\mathrm{H}$ : Interrelation between obesity, oral health and lifestyle factors among Turkish school children. Clin Oral Investig 2011, 15:177-184.

43. de Carvalho Sales-Peres SH, Goya S, de Freitas Sant' Anna RM, Silva HM, Sales-Peres AC, da Silva RP R, de Magalhaes Bastos JR: Prevalence of overweight and obesity, and associated factors in adolescents, at the central West area of the State Sao Paulo (SP, Brazil). Cien Saude Colet 2010, 15:3175-3184.

44. Granville-Garcia AF, de Menezes VA, de Lira PI, Ferreira JM, Leite-Cavalcanti A: Obesity and dental caries among preschool children in Brazil. Rev Salud Publica (Bogota) 2008, 10:788-795.

45. Moreira PVL, Rosenblatt A, Severo AMR: Prevalence of dental caries in obese and normal-weight Brazilian adolescents attending state and private schools. Community Dent Health 2006, 23:251-253.

46. van Gemert-Schriks MCM, van Amerongen EW, Aartman IHA, Wennink JMB, ten Cate JM, de Soet JJ: The influence of dental caries on body growth in prepubertal children. Oral Investig 2011, 15:141-149.

47. Sheller B, Churchill SS, Williams BJ, Davidson B: Body mass index of children with severe early childhood caries. Pediatr Dent 2009, 31:216-221.

48. Cereceda MMA, Faleiros CS, Ormeno QA, Pinto GM, Tapia VR, Diaz SC, Garcia BH: Prevalence of dental decay in elementary school children and association with nutritional status. Rev Chil Pediatr 2010, 81:28-36.

49. Sadeghi M, Lynch CD, Arsalan A: Is there a correlation between dental caries and body mass index-for-age among adolescents in Iran? Community Dent Health 2011, 28:174-177.

50. Jurgensen N, Petersen PE: Oral health and the impact of sociobehavioural factors in a cross sectional survey of 12-year old school children in Laos. BMC Oral Health 2009, 9:29.

51. Juarez-Lopez MLA, Villa-Ramos A: Caries prevalence in preschool children with overweight and obesity. Rev Invest Clin 2010, 62:115-120.

52. Pinto $\mathrm{A}$, Kim $\mathrm{S}$, Wadenya $\mathrm{R}$, Rosenberg $\mathrm{H}$ : Is there an association between weight and dental caries among pediatric patients in an urban dental school? A correlation study. J Dent Educ 2007, 71:1435-1440.

53. Human Development Reports.: http://hdr.undp.org/en/.

54. Tramini P, Molinari N, Tentscher M, Demattei C, Schulte AG: Association between caries experience and body mass index in 12-year-old French children. Caries Res 2009, 43:468-473.

55. Jamelli SR, Rodrigues CS, de Lira PI: Nutritional status and prevalence of dental caries among 12-year-old children at public schools: a casecontrol study. Oral Health Prev Dent 2010, 8:77-84.

56. Scheutz F, Matee MI, Poulsen S, Frydenberg M: Caries risk factors in the permanent dentition of Tanzanian children: a cohort study (1997-2003). Community Dent Oral Epidemiol 2007, 35:500-506.

57. Cinar $A B$, Christensen LB, Hede B: Clustering of obesity and dental caries with lifestyle factors among Danish adolescents. Oral Health Prev Dent 2011, 9:123-130.

58. Acs G, Shulmann R, Ng MW, Chussid S: Effect of nursing caries on body weight in a pediatric population. Pediatr Dent 1992, 14:302-305.

59. Alvarez J: Nutrition, tooth development, and dental caries. Am J Clin Nutr 1995, 61 (supp):410S-416S.

60. Psoter W, Reid B, Katz R: Malnutrition and dental caries: A review of the literature. Caries Res 2005, 39:441-447.

61. Al-Hosani E, Rugg-Gunn A: Combination of low parental educational attainment and high parental income related to high caries experience in pre-school children in Abu Dhabi. Community Dent Oral Epidemiol 1998, 26:31-659.

62. Hooley M, Skouteris H, Millar L: The relationship between childhood weight, dental caries and eating practices in children aged 4-8 years in Australia, 2004-2008. Pediatr Obes 2012, 7(6):461-70. doi:10.1111/j.20476310.2012.00072.x. Dec.

63. World Health Organisation: Obesity and overweight.: http://www.who.int/ mediacentre/factsheets/fs311/en/index.html.
64. Tripathi S, Kiran K, Kamala BK: Relationship between obesity and dental caries in children- A preliminary study. J Int Oral Health 2010, 2:65-72.

65. International Caries Detection and Assessment System Coordinating Committee: The Rationale and Evidence for the International Caries Detection and Assessment System (ICDAS II):: http://www.scribd.com/doc/77330435/ Rationale-and-Evidence-ICDAS-II-September-11.

doi:10.1186/2046-4053-1-57

Cite this article as: Hooley et al:: Body mass index and dental caries in children and adolescents: a systematic review of literature published 2004 to 2011. Systematic Reviews 2012 1:57.

\section{Submit your next manuscript to BioMed Central and take full advantage of:}

- Convenient online submission

- Thorough peer review

- No space constraints or color figure charges

- Immediate publication on acceptance

- Inclusion in PubMed, CAS, Scopus and Google Scholar

- Research which is freely available for redistribution 